\title{
Targeted sensors for glutamatergic neurotransmission
}

Yuchen Hao ${ }^{1,2,4}$, Estelle Toulmé ${ }^{3,4}$, Benjamin Königi,2,4, Christian Rosenmund 3,4 and Andrew J.R. Plested $1,2,4 \S$

${ }^{1}$ Institute of Biology, Cellular Biophysics, Humboldt Universität zu Berlin, 10115 Berlin, Germany. 2Leibniz-Forschungsinstitut für Molekulare Pharmakologie 13125 Berlin, Germany; 3Institute of Neurophysiology, Charité - Universitätsmedizin Berlin; ${ }^{4}$ NeuroCure Cluster of Excellence, Charité - Universitätsmedizin Berlin, corporate member of Freie Universität Berlin, Humboldt-Universität zu Berlin and Berlin Institute of Health, Charitéplatz 1, 10117 Berlin, Germany

§To whom correspondence should be addressed: andrew.plested@hu-berlin.de

\section{Abstract}

Optical report of neurotransmitter release allows visualization of excitatory synaptic transmission. Sensitive genetically-encoded glutamate reporters operating with a range of affinities and emission wavelengths are available. However, without targeting to synapses, the specificity of the fluorescent signal is uncertain, compared to sensors directed at vesicles or other synaptic markers. We fused the state-of-the-art reporter iGluSnFR to glutamate receptor auxiliary proteins in order to target it to postsynaptic sites. Chimeras of Stargazin and gamma-8 that we named SnFR-Y2 and SnFR-y8, retained function and reported spontaneous glutamate release in hippocampal cells, with apparently diffraction-limited spatial precision. In autaptic neurons on micro island cultures, evoked release could be quantitatively detected at tens of synapses in a field of view whilst evoked currents were recorded simultaneously. These experiments revealed a specific postsynaptic deficit from Stargazin overexpression, resulting in synapses with normal release but without postsynaptic responses. This defect was reverted by delaying overexpression. By working at different calcium concentrations, we determined that SnFR-Y2 is a linear reporter of the global quantal parameters and short term synaptic plasticity, whereas iGluSnFR is not. On average, half of iGluSnFR regions of interest showing evoked fluorescence changes had intense rundown, whereas less than $5 \%$ of SnFR-Y2 ROls did. We provide an open-source analysis suite for extracting quantal parameters including release probability from fluorescence time series of individual and grouped synaptic responses. Taken together, postsynaptic targeting improves several properties of iGluSnFR and further demonstrates the importance of subcellular targeting for optogenetic actuators and reporters. 


\section{Introduction}

Synapses pass information from one neuron to another, but their performance is idiosyncratic and unreliable. It was long recognised from electrophysiological work that isolating single synapses reveals variability that is otherwise lost when population responses are measured. In electrophysiology, separating out individual synaptic responses can be achieved either by reducing release probability (DEL CASTILLO and KATZ, 1954), by blocking all other responses (McAllister and Stevens, 2000), using minimal stimulation (Isaac et al., 1996), or by examining connected pairs of neurons (Vyleta and Jonas, 2014) with time resolution in the ms range. In contrast, optical report of neurotransmission via membrane dyes (Griesinger et al., 2005), calcium dyes (Oertner et al., 2002; Enoki et al., 2009), voltage-sensing dyes (Popovic et al., 2015) or neurotransmitterbinding reporters offers direct spatial resolution of synaptic events, usually with a time resolution about 2 orders of magnitude slower. Quantitation and analysis of the relevant optical signals remains challenging (Helassa et al., 2018; James et al., 2019; Soares et al., 2019).

Synapses are diverse in their composition and size (Jontes and Smith, 2000), and it is assumed that these architectural features correspond to functional differences (Cizeron et al., 2020). In principle, accessing individual synaptic inputs to a neuron in space and time should allow correlation of molecular architecture with functional synapse properties and thus reveal synaptic diversity, but functional properties might be less diverse than synapse composition itself (Farsi et al., 2021). Plastic changes in synaptic responses, generated by sensory input (Gambino et al., 2014), are widely taken to represent learning and memory mechanisms, and must in turn increase diversity. However, synaptic contributions to sensory selectivity seem to depend most on the number of synapses that are activated (Scholl et al., 2020) suggesting that synaptic diversity may not serve immediate functional purposes. Instead, because synapses use small numbers of molecules, and tend to operate randomly (Ribrault et al., 2011), diversity may be needed to provide robustness through redundancy. The lack of reliability of certain synapses may be key to their computational power and role in decision making (Evans et al., 2018). These observations strongly motivate us to develop and test optical tools that can report activity with individual synapse resolution, and with a dynamic range sufficient to discern different modes of release, and failures to release.

Early generations of genetically-encoded reporters of glutamate release relied on FRET (Okumoto et al., 2005; Namiki et al., 2007). The advent of bright, single-wavelength genetically-encoded reporters that directly bind neurotransmitters, such as iGluSnFR, has expanded the scope of optical report of synaptic transmission by speeding up report to the limit of optical microscopy (Marvin et al., 2013). This class of sensor has also been adapted to mature faster, and offer affinity and colour variants (Marvin et al., 2018; Helassa et al., 2018). For the purpose of subcellular 
targeting, the compact sensor architecture of iGluSnFR is easy to transplant into other host proteins.

Fusion of fluorescent probes to proteins with selective, organellar targeting allows their subcellular expression. For example, targeting of probes to synaptic vesicles (such as Synaptophluorin (Miesenböck et al., 1998)) has enabled quantitation of vesicle fusion and recovery with high specificity (Balaji and Ryan, 2007; Chanaday and Kavalali, 2018) albeit with slow off-kinetics (Sankaranarayanan et al., 2000) compared to vesicle fusion. On the other hand, geneticallyencoded sensors of neurotransmitters themselves lack targeting to synaptic sites. Even when signals from the latter are localised to putative dendritic spines, the unknown proximity of the sensor to the release site introduces unwanted variability (Soares et al., 2019), because the glutamate signal diverges rapidly in space and time due to diffusion (Raghavachari and Lisman, 2004). Where spatial confinement of the reporter was achieved by exploiting targeting (Kim et al., 2020) or anatomy (Helassa et al., 2018; James et al., 2019; Duerst et al., 2020), the quantitative improvement of the fluorescent report is striking.

Here we report that fusion of iGluSnFR to the glutamate receptor auxiliary proteins $\mathrm{Y}-2$ or $\mathrm{Y}-8$ produces postsynaptic-targeted sensors with improved spatial resolution. The SnFR-y2 reporter gives a high-contrast signal through enrichment at glutamatergic synapses, and surprisingly also shows more stable activity. Spontaneous and evoked release can be resolved at up to $5 \mathrm{~Hz}$, allowing dissection of the presynaptic contribution to short term plasticity and release parameters in cultured neurons at the single synapse level.

\section{Results}

Several groups have pursued mutagenesis of the Glt1 glutamate binding domain to reduce the affinity of iGluSnFR for glutamate (Helassa et al., 2018; Marvin et al., 2018), with aim of improving its spatial and temporal resolution. We examined several mutants using patch-clamp fluorometry (Fig. S1). A particularly interesting mutant that has not been previously reported is the Y230F substitution that removes a hydroxyl group that coordinates glutamate in the Glt1 domain. This mutation increased $\Delta F / F$, the fractional fluorescence change upon glutamate binding (possibly by reducing fluorescence in the absence of glutamate), and sped the kinetics of the off-response (Fig. S1). As expected, the Y230F mutation also reduced the apparent affinity for glutamate. To assess the kinetics of this mutant in synapses, we turned to autaptic microisland neuronal cultures. In this preparation, a single patch-clamp electrode can produce escaping action potentials that trigger glutamate release from synaptic terminals and record the resulting postsynaptic currents. However, we were surprised to discover that the Y230F mutant gave almost no fluorescent response in 
A

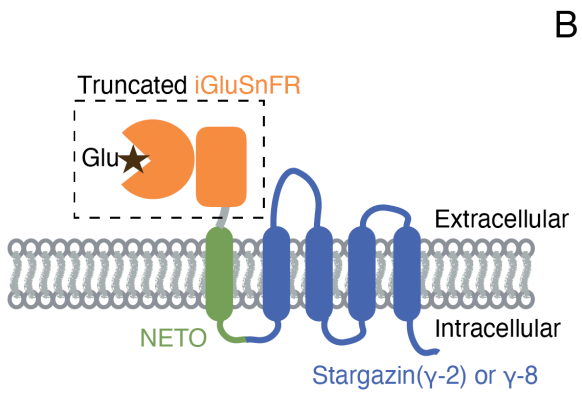

B

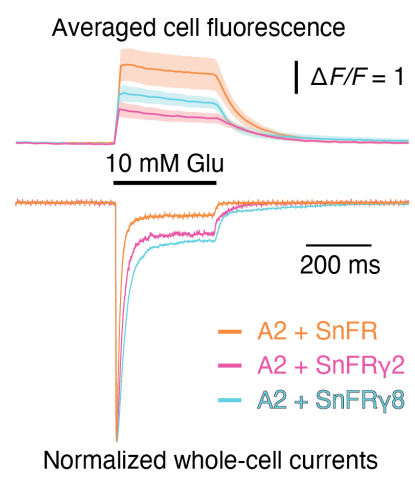

C

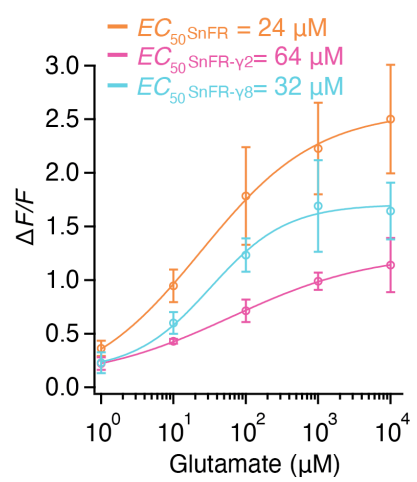

G

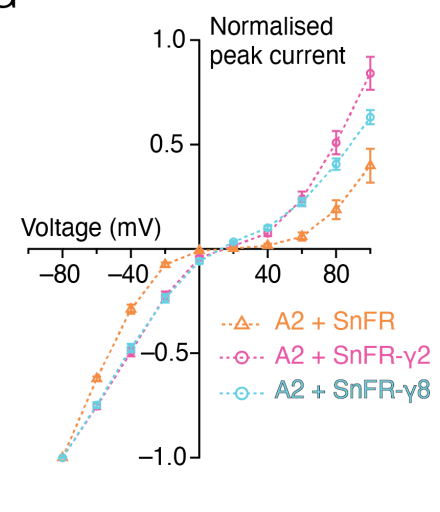

Figure 1. Patch-clamp fluorometry of SnFR-p2 and SnFR-y8 in HEK cells. (A) Schematic view of SnFRү2 and SnFR- $\gamma 8$ chimeras, comprised of the truncated extracellular part of iGluSnFR (orange) to signal glutamate binding (black star), NETO as transmembrane linker (green) and Stargazin ( $\gamma-2)$ or $\gamma-8$ (blue) to act as a postsynaptic anchor. (B) Average fluorescent responses and normalised representative traces of currents for iGluSnFR (orange, $n=15$ ), SnFR- 2 (magenta, $n=8$ ) and SnFR- $\gamma 8$ (cyan, $n=7$ ) during $10 \mathrm{mM}$ glutamate application. GluA2 receptors were co-expressed with iGluSnFR, SnFR-ү2 or SnFR- $\gamma 8$. The current recording from whole-cell patch-clamp fluorometry acts as a fiduciary for membrane expression, normal auxiliary protein function and fast solution exchange. (C) Glutamate concentration-fluorescence response relationships for iGluSnFR $(n=4)$, SnFR- $\gamma 2(n=4)$ and SnFR- $\gamma 8(n=5)$ in HEK cells. (D-E) Statistics of peak fluorescent response and decay time constants (tau) for iGluSnFR $(n=15)$, SnFR- $\gamma 2(n=$ 8) and SnFR- $\gamma 8(n=7)$, recorded as in panel (B). (F-G) Steady-state currents (normalised to peak response) and $I-V$ relations for peak currents elicited by $10 \mathrm{mM}$ glutamate for GluA2 and iGluSnFR cotransfection $(n=$ $6)$, and for SnFR-y2 $(n=10), \operatorname{SnFR}-\gamma 8(n=10)$ complexes. Error bars represent standard deviation of the mean. Probability of no difference from Student's $t$-test: ${ }^{*} p<0.05 ;{ }^{* \star *} p<0.001,{ }^{* \star \star \star} p<0.0001$.

autaptic hippocampal neurons following stimulation (Fig. S2), whereas in the same cultures, the regular iGluSnFR gave robust fluorescence responses, phase-locked to the postsynaptic currents, as did Synaptophluorin (with about 100x slower off kinetics). Paradoxically, the Y230F was expressed on the neuronal surface and responded robustly to $10 \mathrm{mM}$ glutamate perfusion over the autapse (Fig. S2). Combined with the observation that the fluorescence from unmodified iGluSnFR on average occurs over a spatial extent much larger than an individual synapse $\left(\sim 10 \mu \mathrm{m}^{2}\right.$, see below) this observation led us to hypothesise that the low affinity sensor was on average not sufficiently close to the synaptic glutamate signal to properly report it. Other low affinity sensors 
have been reported (Helassa et al., 2018; Marvin et al., 2018) but as outlined above, their localisation in relation to postsynaptic densities (and therefore the signal they report) is uncertain.

iGluSnFR is membrane targeted through a single pass pDisplay peptide (Marvin et al., 2013), which provides no localisation signal. To address this aspect, we fused the iGluSnFR extracellular domain to AMPA-type glutamate receptor in order to target it to excitatory synapses. Inline fusion at a permissive site in AMPA receptor subunits GluA1 and GluA2 extracellular region produced a sensor with a very small $\Delta F / F$ (Supplementary Fig. 3). Instead, we turned to the auxiliary proteins (TARPs) that decorate the periphery of AMPA receptors. To place the iGluSnFR sensor domain on the extracellular side of the membrane we fused a single pass transmembrane helix (from the NETO2 kainate receptor auxiliary protein (Zhang et al., 2009) to avoid any adventitious competition against the AMPA receptor - TARP interaction) to the $\mathrm{N}$-terminus of Stargazin ( $\mathrm{\gamma}-2)$ or $\mathrm{\gamma}-8$ (Fig. 1A). These chimeric sensors had similar performance to the original iGluSnFR and associated normally with AMPA receptor complexes in HEK cells (Fig. 1), also acting to modulate AMPA receptor gating. We named these reporters SnFR-Y2 and SnFR-Y8.

We made adeno-associated viruses (AAVs) of both SnFR-Y2 and SnFR-ү8. Bulk hippocampal cultures infected with these viruses revealed regular fluorescence spikes (typically about $1 \mathrm{~Hz}$ frequency, see Fig. 2 and Supplementary Movie 1), whereas the AAV of iGluSnFR gave wide, diffuse and, in our hands, relatively infrequent fluorescent signals (Fig. 2 and Supplementary Movie 2). At hand-picked regions of interest, SnFR-y2 and SnFR-y8 showed a more localised signal (Fig. 2). To assess if the locus of expression was indeed synaptic, we co-infected the cultures with a lentivirus for Homer-tdTomato. The complexity of the SnFR-y2 signal between intracellular and synaptic sites forbade a simple measure of colocalization, but responsive SnFR-y2 spots were clearly enriched at Homer positive spots, presumably corresponding to synaptic connections (Fig. $3 A)$.

Background subtracted heat maps revealed a sharper signal for SnFR-Y2 compared to iGluSnFR (Fig. 3B). Taking a line profile through the centre of the peak response showed that the apparent half-width of the responses during spontaneous neurotransmission was on average less than $500 \mathrm{~nm}$ (Figs. 3D \& E) or about 3-fold narrower than for iGluSnFR in our cultures. When averaging across frames, the half width at some sites was of the order of $300 \mathrm{~nm}$, or in other words, probably limited by diffraction of the microscope (Fig. 3D). For this spontaneous neurotransmission, both the peak amplitude and the mean amplitude from the peak and subsequent 2 frames from SnFR-Y2 was slightly increased compared to iGluSnFR (Fig. 3F \& G), possibly because SnFR-y2 and SnFR-y8 experienced a higher degree of saturation than their non-targeted version (see Discussion). 
A

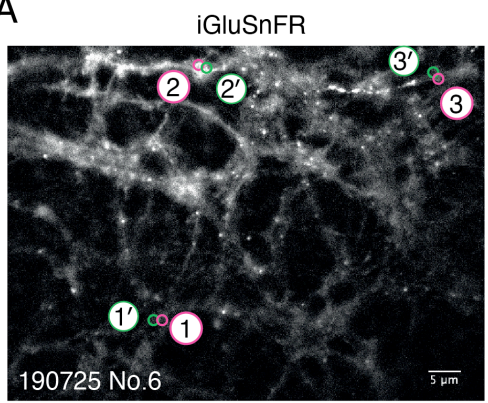

B
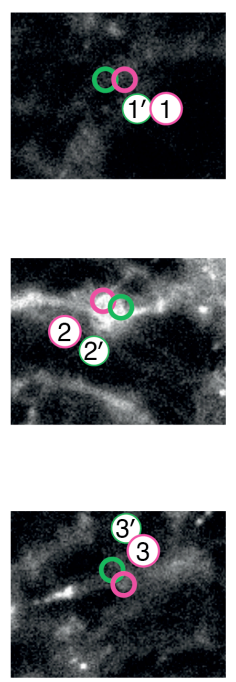

C

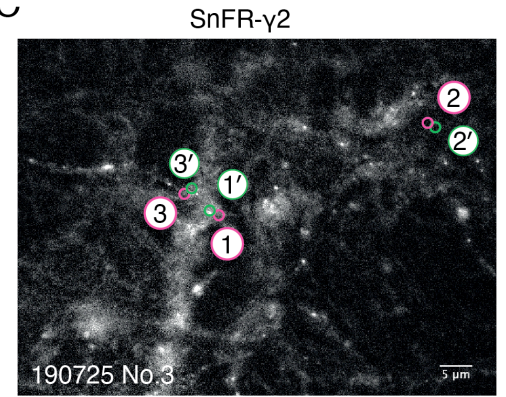

$\mathrm{D}$
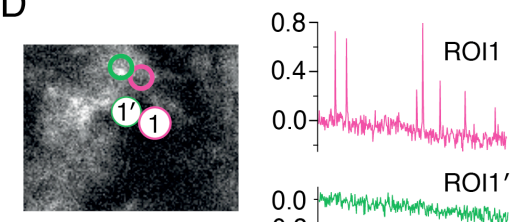

0.0
-0.2
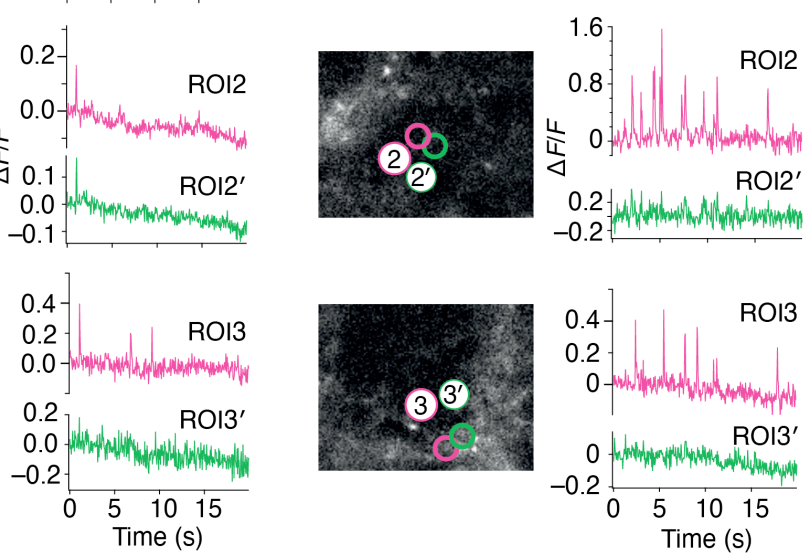
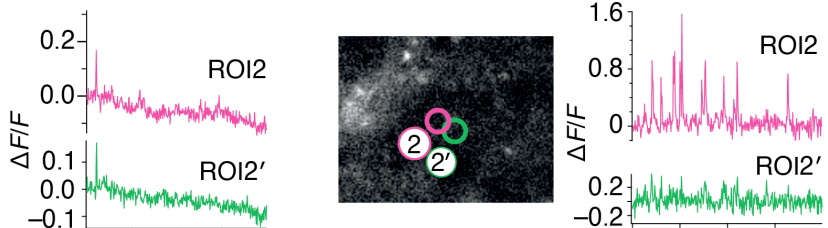

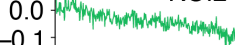

-0.2 .

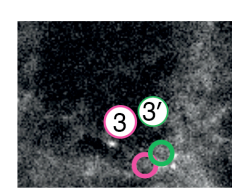

$\mathrm{E}$

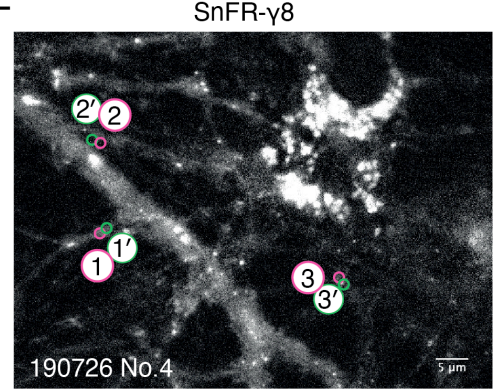

$\mathrm{F}$
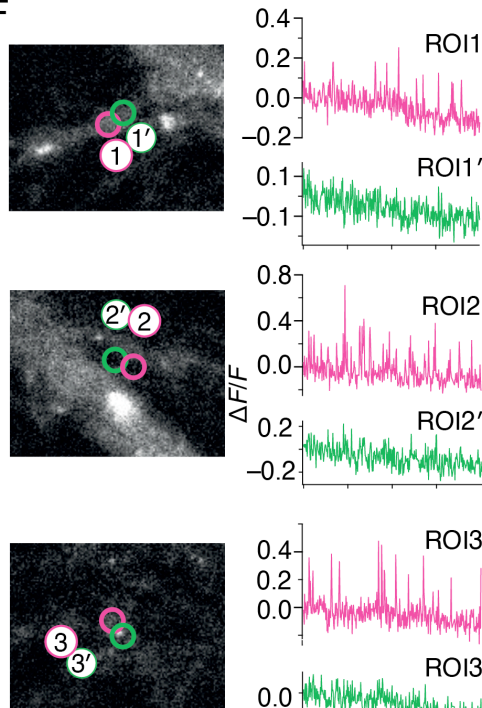

Figure 2. Spontaneous responses of iGluSnFR, SnFR-ү2 and SnFR- $\gamma 8$ expressed in cultured rat hippocampal neurons. (A) Fluorescence micrograph of neurons expressing iGluSnFR with 3 typical regions of interest marked (ROls, indicated as magenta circles) and compared with adjacent ROls of the same size (shown as green circles, with prime). Scale bar, $5 \mu \mathrm{m}$. (B) Higher magnification views (2.2-fold zoom) around the individual ROls and the corresponding color-coded fluorescence time series. Vertical deflections correspond to spontaneous responses. Note similarity between magenta and green traces). All fluorescence time series were collected at $20 \mathrm{~Hz}$. (C \& D) As in panels A \& B, but for neurons expressing SnFR- 2 2. Note the lack of features in the green traces. $(E \& F)$ As in panels $A \& B$, but for neurons expressing SnFR- $\gamma 8$.

Infection of autapses with SnFR-Y2 and SnFR-Y8 gave punctate signal in the majority of cases, but for some neurons, the signal remained diffuse. Although not obviously related, we also regularly measured a near absence of evoked neurotransmission (Fig. 4B) in about half the autaptic neurons following infection before DIV 3. Robust evoked glutamate release (as reported by fluorescence changes from the sensors) was present, and we failed to detect any change in VGLUT puncta size or number on MAP2 positive neurites in immunostaining experiments (Fig. S4), when comparing to non-infected neurons or neurons infected with iGluSnFR. These observations suggested a profound postsynaptic deficit due to overexpression of SnFR- $\mathrm{Y} 2$ and SnFR-y8. We reasoned that, given that cultured neurons are still developing in this time window, we could infect the neurons later (after 6 days in vitro, DIV) and recover the evoked currents. 
A

A Spontaneous release
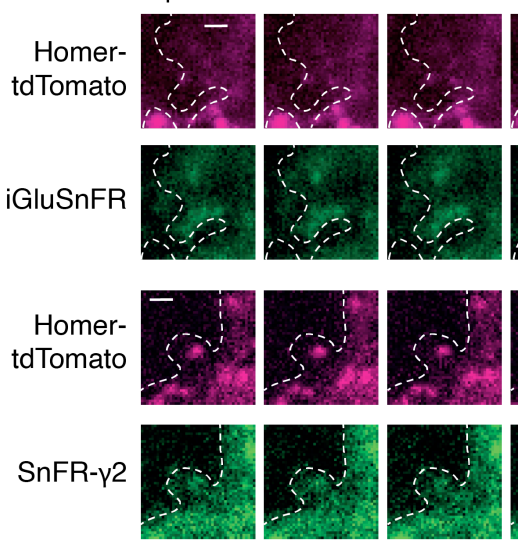

$25 \mathrm{~ms}$ frames
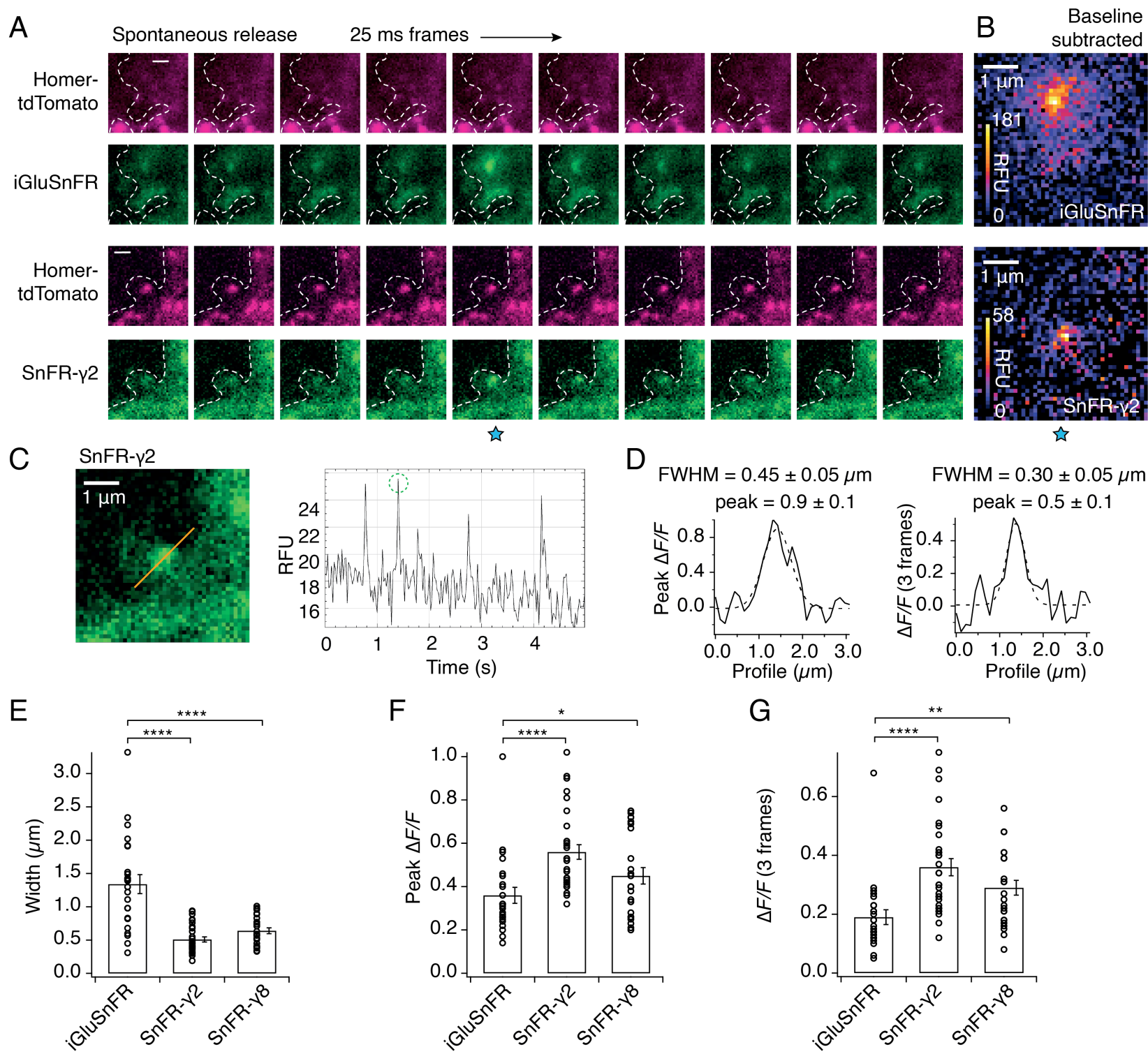

Figure 3. SnFR-y2 and SnFR-y8 give a more spatially precise signal than iGluSnFR. (A) Time-lapse with $40 \mathrm{~Hz}$ frame rate of representative spontaneous fluorescence responses for iGluSnFR and SnFR-ү2 (separate recordings from separate neurons). For SnFR-y2, the signal coincides with Homer. The cell boundary (dashed line) was drawn by hand. Scale bars, $1 \mu \mathrm{m}$. (B) Background-subtracted heat maps of spontaneous fluorescence signals from the frame marked with the blue star. (C) Line profile (orange) and mean fluorescence time series from this line profile over 5 seconds. The second peak response was chosen for line profile and width analysis. (D) Fluorescence line profiles and Gaussian fits (dashed line) of individual peak response and average of the peak and the 2 consecutive frames. The widths of the fitted Gaussian profiles were 0.45 and $0.30 \mu \mathrm{m}$ for individual peak and average 3 frames, respectively. (E) Fitted width profiles for SnFR-Y2 ( $n=30,5$ neurons) and SnFR-ү8 ( $n=25,6$ neurons) are substantially narrower than SnFR ( $n=24,8$ neurons). (F \& G) SnFR-Y2 $(n=30)$ and SnFR-ү8 $(n=25)$ show larger relative fluorescence changes than SnFR $(n=24)$ with either individual peak or average 3 frames. Data represent single fluorescent spots and mean \pm standard deviation of the mean. Probability of no difference was from Student's t-test as follows: ${ }^{*} P<0.05 ;{ }^{* *} P<0.01,{ }^{* * *} P<0.0001$. 


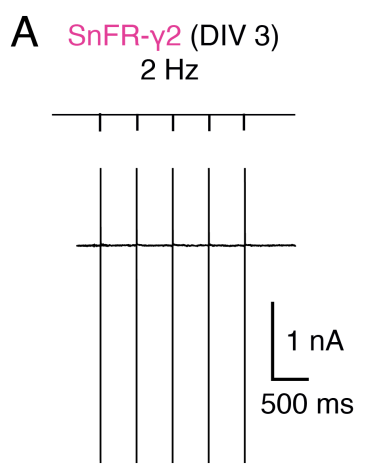

iGluSnFR (DIV 3) $2 \mathrm{~Hz}$
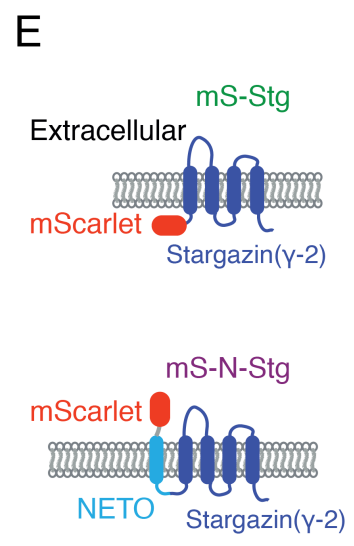
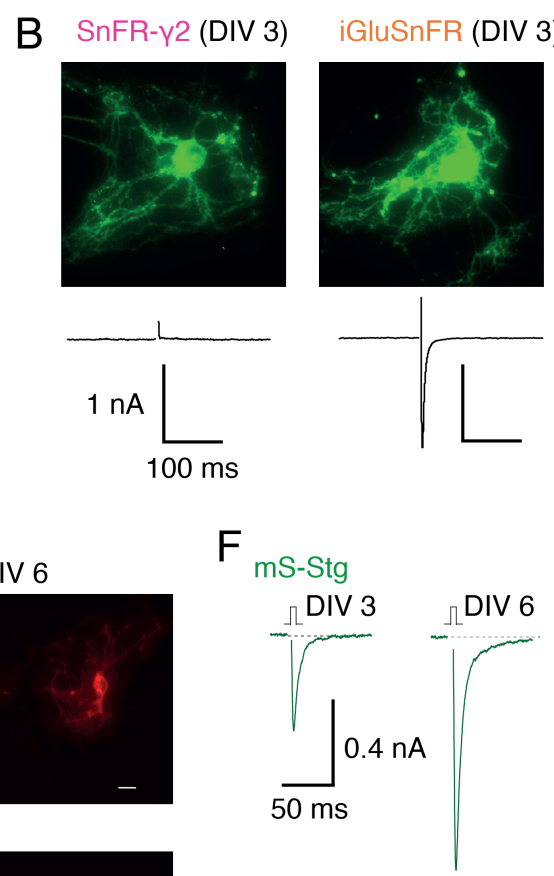

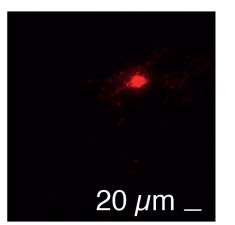

DIV 6
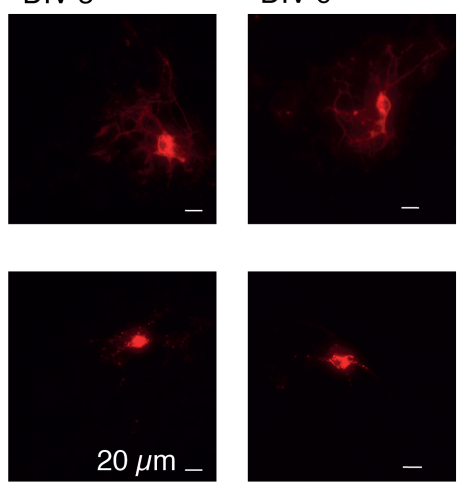

mS-N-Stg

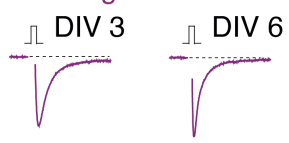

C

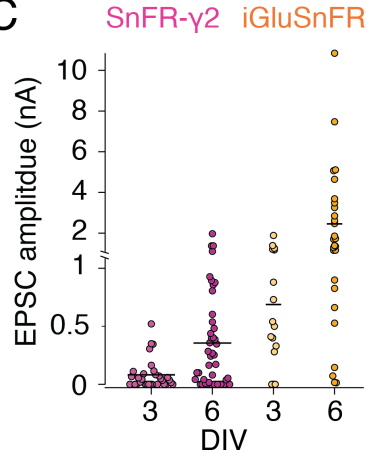

G

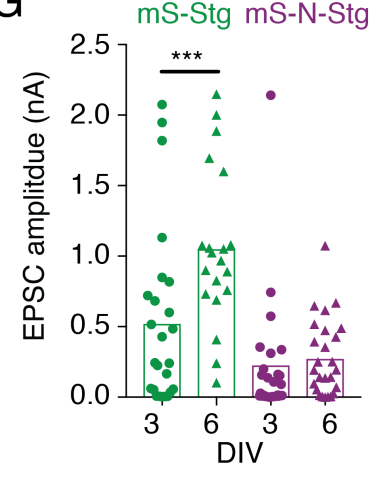

Figure 4. Early infection with SnFR-y2 or Stargazin disrupts glutamatergic currents in autaptic hippocampal neurons. (A) Responses of autaptic neurons infected with SnFR- $\gamma 2$ or iGluSnFR to a five pulse train at $2 \mathrm{~Hz}$. (B) The maximum projections of the fluorescence movies collected from each cell in panel $(A)$, and the first response in the train with the action potential partially blanked. Both cells showed fluorescent responses to $2 \mathrm{~Hz}$ stimulation (not shown), but only the iGluSnFR infected cell showed an EPSC. (C) Summary graph of the EPSC amplitudes in autaptic hippocampal neurons expressing SnFR- $\gamma 2$ or iGluSnFR, infected on DIV 3 or before, and DIV 6 or after. (E) Cartoon of Stargazin constructs tagged on the $\mathrm{N}$-terminal with mScarlet (mS-Stg, green, upper row) or extracellular mScarlet with NETO2 helix (mS-N-Stg, magenta, bottom row) and representative fluorescence images of autaptic hippocampal neurons infected at DIV3 (left pair) or DIV6 (right pair). (F) Representative excitatory postsynaptic currents (EPSC) evoked by one action potential in autaptic hippocampal neurons expressing mS-Stg (green traces) or mS-N-Stg (magenta traces) infected at DIV3 (left trace) or DIV 6 (right trace). (G) Summary graph of the EPSC amplitudes in autaptic hippocampal neurons expressing mS-Stg (green) or mS-N-Stg (magenta) at either DIV3 (circles) or DIV6 (triangles) ( $n=25,24,20$ and 26 respectively). Data shown are individual points, bars are means. Three independent neuronal cultures were examined, with recording and analysis performed blind. Probability of no difference was from the Mann-Whitney non-parametric test $\left.{ }^{\star \star \star \star} p<0.001\right)$.

Indeed, a majority of autaptic neurons retained an appreciable evoked response following late infection (neurons giving EPSC > 100 pA: 7 of 31, DIV 3 infection and 24 of 45, DIV 6; Fig. 4C). The fluorescent signal and presynaptic markers (Fig. S4) were unaffected by the late infection. Given that infection with iGluSnFR does not affect evoked currents much (Fig. 4C), we reasoned that auxiliary proteins in overexpression might be causing the postsynaptic deficit. Indeed, early infection (before DIV3) of either of two Stg constructs tagged with mScarlet (with or without the 
fused N-terminal NETO transmembrane section) were highly deleterious to evoked currents in autapses (Figs. 4F \& G). Miniature currents were also strongly reduced in amplitude and frequency, to the point that we failed to detect minis in a large proportion of cells (Fig. S5), whereas paired-pulse ratio (20 ms interval) was unaffected. However, a late infection (after DIV6) had much less impact, suggesting a previously unheralded developmental pathology at the postsynapse from early Stargazin overexpression.

In autaptic neurons infected with AAVs at DIV6, the signal from iGluSnFR in response to evoked transmission at $5 \mathrm{~Hz}$ was again more diffuse than the response of SnFR-y2 (Fig. 5). We selected small regions of interest and adjacent neighbour regions, again by hand. Whilst neighbour and principal fluorescence time series usually matched well for iGluSnFR (Fig. 5C), for most ROls in the SnFR-Y2 cells, the neighbour region was silent (Fig. 5F).

Having established these prerequisites, we returned to our original motivation for improving the spatial and temporal response of iGluSnFR, which was to evaluate synaptic transmission at individual connections. We recorded movies of autaptic neurons infected with iGluSnFR (Supplementary Movie 3), SnFR-Y2 (Supplementary Movie 4) or SnFR-Y8. At the same time, we subjected the neurons to trains of 10 stimuli at $5 \mathrm{~Hz}$ in $0.5 \mathrm{mM}, 2 \mathrm{mM}$ and $4 \mathrm{mM}$ calcium. Escaping action potentials were generated from short depolarising pulses (see Methods), and we recorded the evolved postsynaptic currents under voltage clamp. From these movies, we examined the simultaneous evoked fluorescence responses of individual ROls.

Rather than choosing regions of interest by eye, we employed a custom-written imageJ macro to systematically scan movies ( $25 \mathrm{~Hz}$ frame rate) for ROls that show similar statistics to the expected responses. Responses at $2 \mathrm{mM}$ calcium were most reliable across the different sensors and we used these to identify ROls with $>20$ positive-going fluorescence transients per movie in which we made 50 stimulations. Composite ROls were constructed from the contiguous regions identified. Strikingly, but perhaps unsurprisingly, the ROls found in this way (Fig. 6) were much smaller on average for SnFR-Y2 (mean areas: $2.5 \mu \mathrm{m}^{2}$ vs $9.6 \mu \mathrm{m}^{2}$ ) than for iGluSnFR. On average, neurons infected with iGluSnFR gave about twice as many ROls as either SnFR-Y2 or SnFR-Y8 (Fig. 6C; 207 ROls for iGluSnFR, 107 for SnFR-y2 and 97 for SnFR-y8, each over 6 neurons). For all but one neuron, the number of ROIs from SnFR-y8 was too few to pursue a further analysis. 

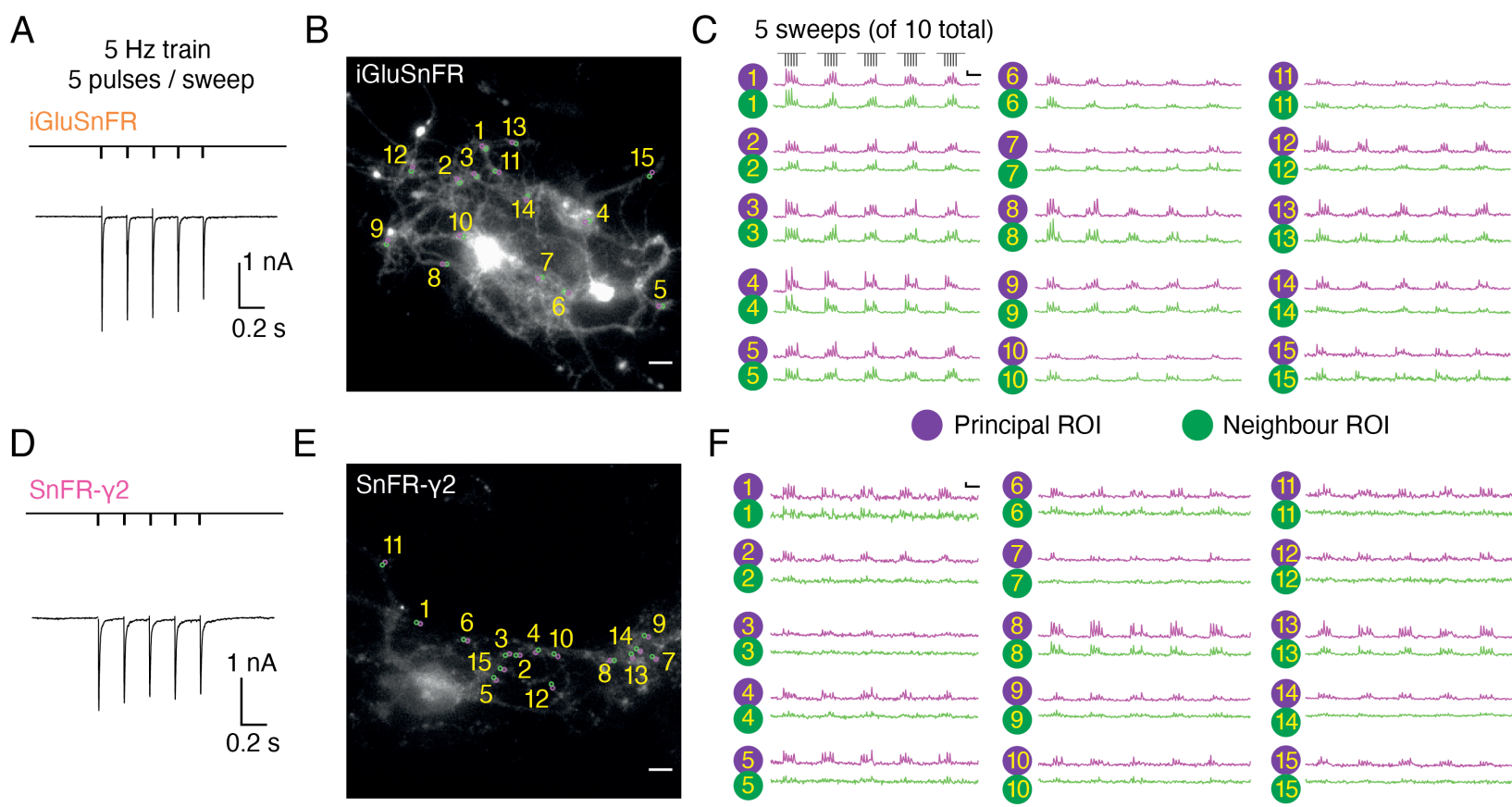

Principal ROI

Neighbour ROI
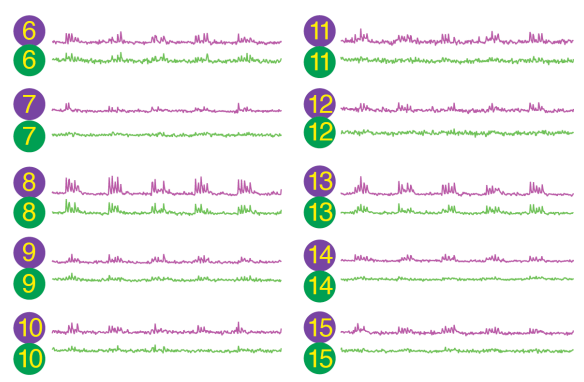

Figure 5. Evoked currents and fluorescent responses of iGluSnFR and SnFR-y2 from autaptic neurons. (A) A train of 5 action potentials (APs) evoked 5 glutamatergic currents (EPSCs) from an autaptic neuron infected with iGluSnFR (infected after DIV 6) in $2 \mathrm{mM}$ calcium. The depolarisation and single AP are blanked to reveal the EPSC. This stimulus was repeated 10 times. (B) Fluorescence micrograph of the autapse recorded in (A) with 15 principal ROls (purple circles) against neighbouring ROls of the same size (green circles). Scale bar, $5 \mu \mathrm{m}$. (C) Fluorescence time series from the principal and neighbour ROls for 5 sets of stimuli (indicated above trace 1). Note similarity between purple and green traces in each case, indicating a broad signal. Scale bar $\Delta F / F$ is 0.2 and timebase $1 \mathrm{~s}$. (D-F) Equivalent data from an autapse expressing SnFR-Y2 (infected after DIV 6). Note that most green traces from neighbour ROIs do not respond (ROls 8 and 13 are exceptions). Scale bars match those in panels (B) and (C).

Although iGluSnFR gave more ROls, when we analysed the peak responses from each ROI, we saw that the responses to iGluSnFR tended to run down (assessed as the magnitude of the last $10 \%$ of responses to the first $10 \%$, Fig. $6 \mathrm{E}$ ). Indeed at least half of automatically-identified ROIs from the iGluSnFR movies showed rundown greater than 50\% (Fig. 6F), whereas less than $7 \%$ of ROls selected from SnFR-y2 cells did. There was no relation between the size of the compound $\mathrm{ROI}$ and the extent of rundown (Fig. 6G).

To understand if the rundown was a problem of the sensor, or perhaps simply an accurate report of the neurotransmission in these cells, we looked at how well the responses of the fluorescence reporters followed the summed postsynaptic electrical response to AP-induced release of glutamate, which we measured simultaneously. Postsynaptic currents in iGluSnFR and SnFR-Y2 showed similar short-term plasticity (Fig. 7A \& B). With $2 \mathrm{mM}$ calcium, the peak amplitude exhibited mild depression during trains of 5 pulses. The initial amplitude was larger and showed a stronger reduction at $4 \mathrm{mM}$ calcium, consistent with multivesicular release and vesicle depletion, whereas responses were small and did not change during the train for $0.5 \mathrm{mM}$ calcium. We described the 
A

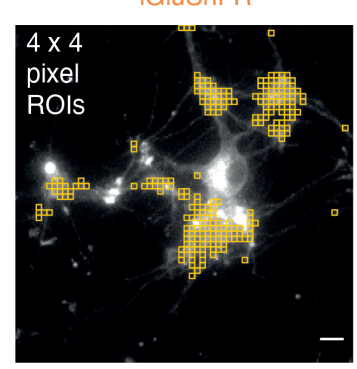

B

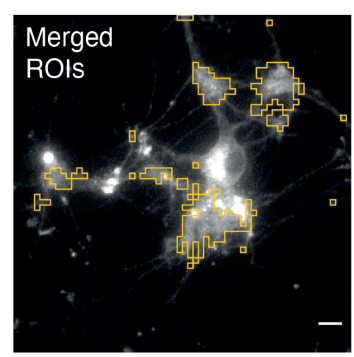

$\mathrm{E}$

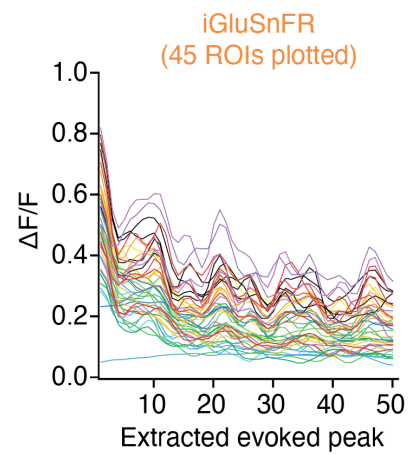

SnFR-y2
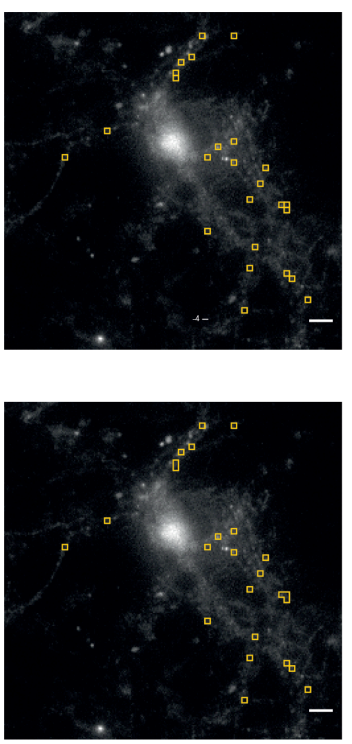

SnFR-y2

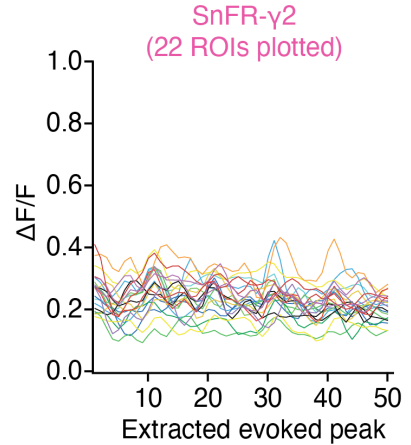

SnFR-ү8
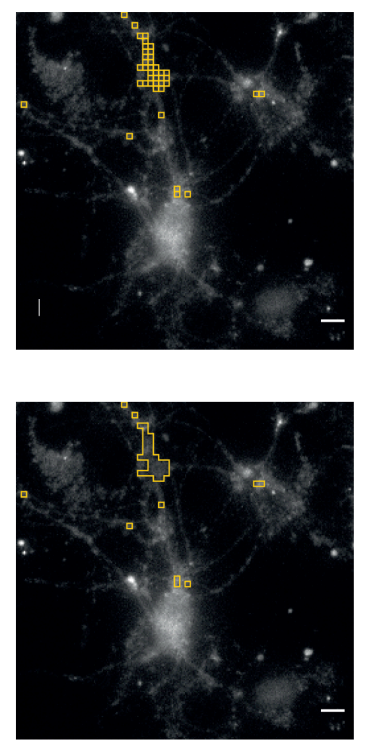

$\mathrm{F}$

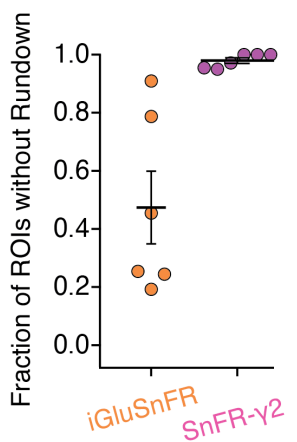

C

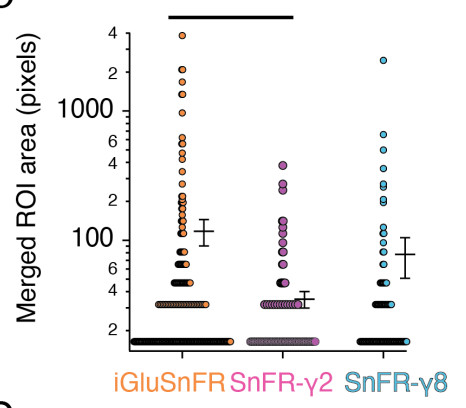

D

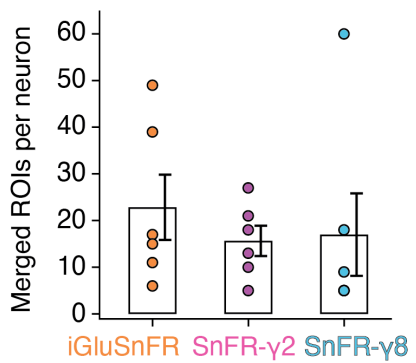

G

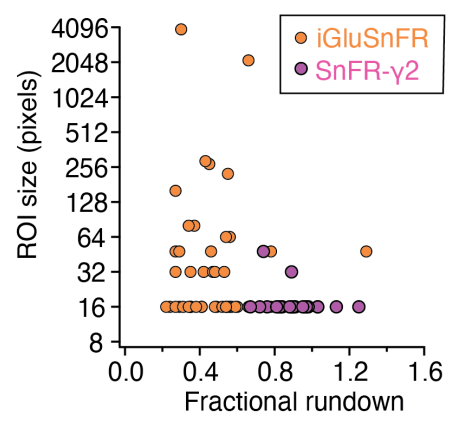

Figure 6. Targeted sensors giver fewer, smaller and more reliable ROls (A) ROI size analysis for evoked fluorescent responses of SnFR, SnFR- $\gamma 2$ and SnFR-y8 on autaptic neurons. ROls (orange outlines) automatically selected by a custom-written Fiji script (supplementary files) fulfilling the following conditions: $4 \times 4$ pixel ROI, intensity increase for each event $\geq 10 \%, S N R \geq 3$, and number of expected peaks in a movie $>20$. Scale bars, $5 \mu \mathrm{m}$. (B) As panel A but with contiguous ROls merged. (C) Statistics of ROI sizes after merging for SnFR, SnFR-y2 and SnFR-y8 for 6 cells in each case. SnFR- $\gamma 2$ shows fewer ROls and smaller ROIs than SnFR (probability of no difference from Student's t-test was 0.0016). (D) On average, SnFR- 22 and SnFR-y8 produced fewer ROIs per neuron (single field of view, 60x objective). (E) The smoothed amplitudes of 50 peaks extracted from movies of two neurons, one expressing iGluSnFR and one expressing SnFR-y2. 45 ROIs were extracted for SnFR and 22 for SnFR-y2. Stimulation was 10 groups of 5 action potentials at $5 \mathrm{~Hz}$. (F) Summary over cells included in analysis. On average, more than $50 \%$ of SnFR ROls were prone to rundown whereas SnFR-y2 ROls were not. (G) Rundown of SnFR responses was unrelated to the size of the automatically-determined compound ROI. Error bars represent standard deviation of the mean. 
B
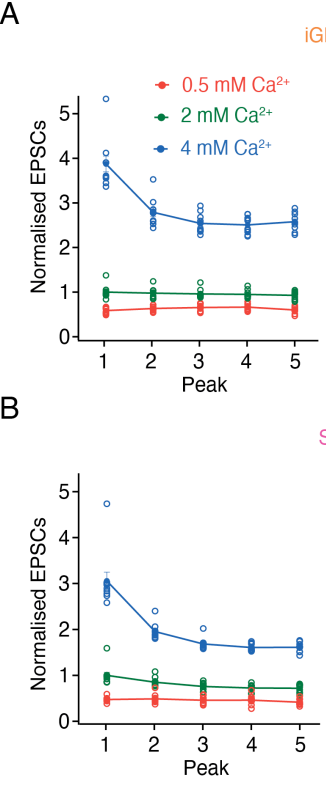

$\mathrm{D}$

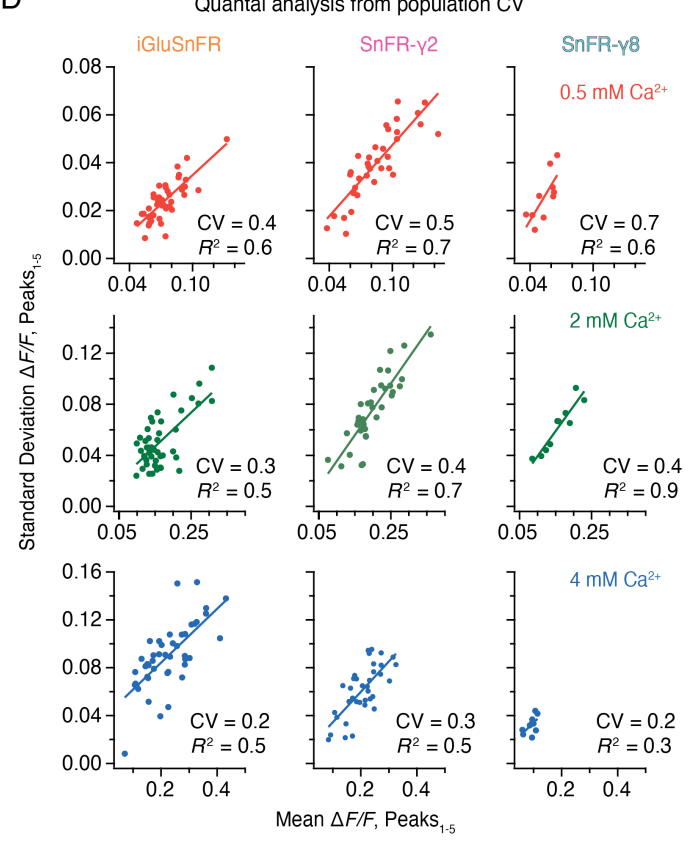

C
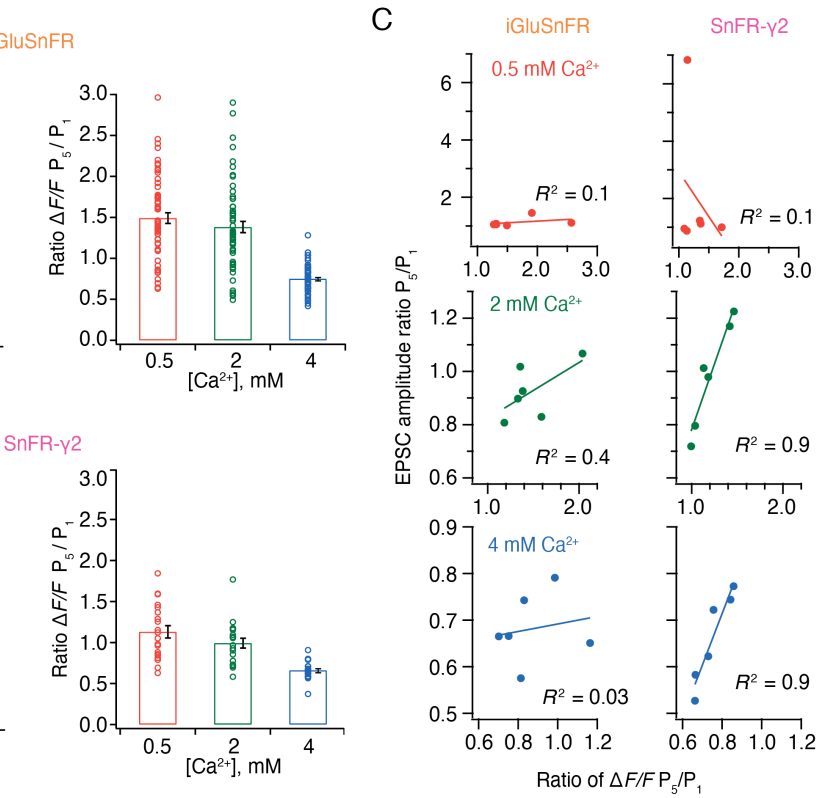

$\mathrm{E}$

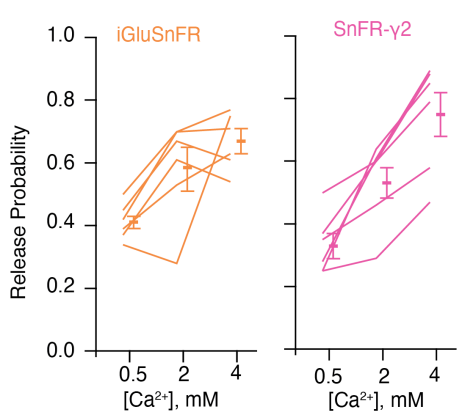

$F$

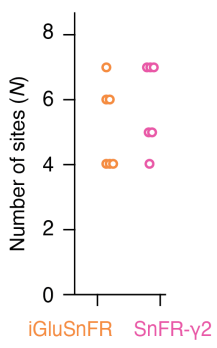

Figure 7 Correlations and variance of fluorescence responses (A) Short term plasticity of a train of 5 EPSCs evoked at $5 \mathrm{~Hz}$ for a single neuron (left, single responses to 10 trains and mean, normalised to the amplitude of the first response in $2 \mathrm{mM}$ calcium). $\Delta F / F$ for multiple regions of interest in the same cell (right panel, bar shows mean and standard deviation of the mean). (B) As panel A but for a cell expressing SnFRү2. (C) Correlation between short term plasticity (ratio between responses to 5th and 1st stimulus) from electrophysiology and fluorescence. (D) The mean fluorescence response and the standard deviation of these responses from individual ROls from one cell, across 10 groups of 5 stimuli at $5 \mathrm{~Hz}$ is plotted at the three different calcium concentrations for the three different constructs. The coefficient of variation (CV, slope of the curve) was used in (E) to estimate population quantal parameters. (E) Release probabilities estimated over the population of responses. Note that the increase in release probability was always monotonic for SnFR-y2 but not for iGluSnFR. (F) The number of release sites used in the calculations was poorly determined. 
degree of depression with the ratio of amplitudes in response to the first and last stimulation in the group (Amplitude 5 / Amplitude 1 ). For fluorescence responses, we obtained peak responses from ROI time series following automatic (but supervised) background subtraction and discarded ROls with a low peak signal to noise ratio (>3, see Methods).

In movies recorded from iGluSnFR expressing cells, there was no relation between the depression of the postsynaptic AMPA receptor response and the average depression of the fluorescence signal over the ROIs (Fig. 7C). In contrast, there was an excellent correlation between the SnFRY2 depression and the postsynaptic current depression at 2 and $4 \mathrm{mM}$ calcium (Fig. 7C) indicating that the synaptic release reported by SnFR- $\gamma 2$ across tens of sites per neuron accurately represents the average synaptic response to glutamate release. The lack of correlation of signals from iGluSnFR is likely because of rundown and also possibly because indistinct localisation leads it to report stray glutamate as well as synaptic glutamate, particularly in conditions where repeated glutamate release occurs (5 $\mathrm{Hz}$ or above, see Discussion).

Taking each train of 5 responses, we could plot the standard deviation of the 5 responses against the mean. In this way, we could estimate the coefficient of variation (CV) for a large number of synapses and responses (Fig. 7D). Quantal analysis (DEL CASTILLO and KATZ, 1954) suggests that the square of the $\mathrm{CV}$ is a simple function of the number of sites and the probability of release (see Methods). Taking the slopes from linear fits to paired SD/mean values, we performed a nonlinear fit to the estimated squared CV values across the whole population, allowing for a different release probability at each calcium condition and common $N$. Even though $N$ must be averaged over a large number of sites, we fixed $N$ to be an integer to facilitate the search.

For CV analysis, the release probability estimated from SnFR-Y2 increased monotonically with $\mathrm{Ca}^{2+}$ concentration, as expected (6/6 cells) and gave plausible values. By contrast, the release probability estimated from iGluSnFR responses did not increase monotonically for most cells (Fig. $7 \mathrm{E}$ ). For both conditions, $N$ was hard to determine ( 3 data points but 4 unknowns) and the data were often fit equally well with a range of $N$, which took values from 4-8. Previous analysis on connected cultured hippocampal neurons using electrophysiology gave similar values $(N=5$ and $P_{\mathrm{R}}=0.4$ from CV analysis (Bekkers and Stevens, 1990) to the ones that we obtained from SnFR-Y2 at $2 \mathrm{mM} \mathrm{Ca}^{2+}$.

Given these encouraging results, we next asked if SnFR-y2 could be used to measure the quantal properties of individual synapses, and if it offered any advantages over the non-targeted iGluSnFR. To do this, we performed automated baseline subtraction from each ROI and detected peak responses according to a manually determined pattern from the mean of all responses. For both 


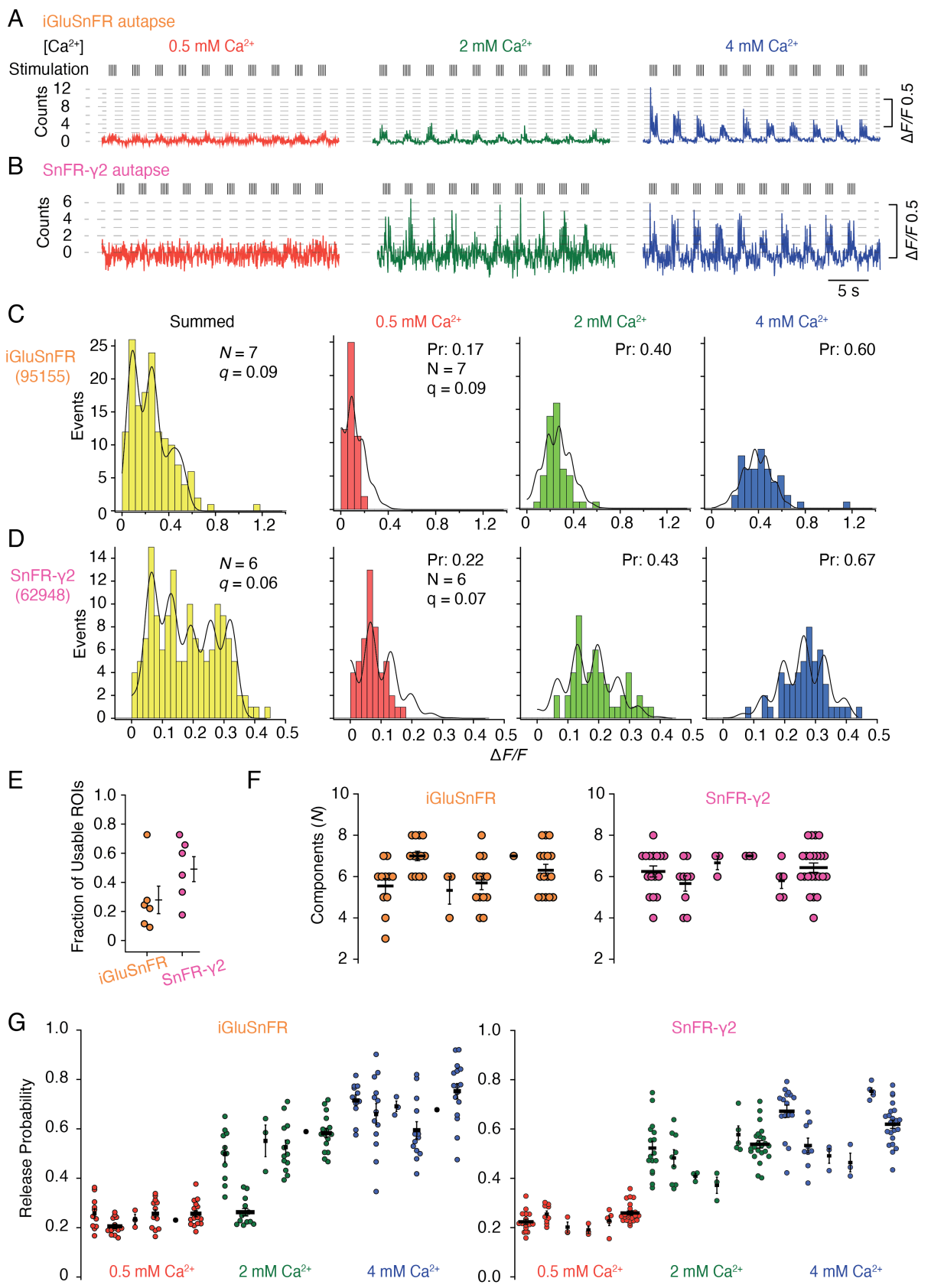

Figure 8 Quantal analysis in autaptic neurons (A) Background subtracted fluorescence time series from one ROI from an iGluSnFR-expressing neuron at different calcium concentrations. Nominal quantal levels are indicated by dashed lines. The first response in $4 \mathrm{mM}$ calcium is disproportionately large (and was usually ignored), and that steady-state responses accumulate during each train. (B) As in (A) but for a cell expressing SnFR- $\gamma 2$, which showed smaller but more consistent signals. A detailed analysis of these two time series and others recorded in parallel is in Fig. S6. (C) Histograms of peak fluorescence responses from the iGluSnFR ROI in panel A. The summed histogram (yellow) represents 150 pooled responses, fitted with a mixed Gaussian model. Other histograms were separately fitted with the binomial model, where the quantal size (here $\Delta F / F=9 \%$ ) was globally optimized, with the scale. The release probabilities $(\mathrm{Pr})$ were optimised for each calcium concentration. The number of release sites $(N=7)$ was chosen to give the best fit (highest Kolmogorov-Smirnoff probability). (D) As panel C but for the SnFR- $ү 2$ ROI. (E) Only 25\% of ROls from the total were suitable for histogram fitting for iGluSnFR, whereas on average half of the SnFR- $\gamma 2 \mathrm{ROI}$ were useable. (F) The distributions of $N$ chosen to give the best fit, from all the useable ROls from each cell for iGluSnFR and SnFR-ү2 (6 cells each). (G) The distributions of release probability for each ROI, grouped by cell and calcium condition. 
iGluSnFR and SnFR-Y2, we observed fluorescence time series with clear calcium dependence and quantal behaviour (Fig. S6). From 50 stimulations at each concentration of calcium (150 events in total), at about half of the ROls from iGluSnFR we could build histograms and fit globally (see Fig. S7 for workflow). For SnFR-y2, 80\% of the automatically ROI were usable for quantal analysis, and these ROls reported a range of release probabilities similar to those determined from the CV analysis (Fig. 8). In the majority of ROls, according to the Kolmogorov-Smirnoff test for the goodness of fit, the Binomial model gave a better fit than the Poisson model (Bhumbra and Beato, 2013; Malagon et al., 2016). Examples of imperfect fits are shown in Fig. S8. For SnFR-Y2, the distributions of peak amplitudes gave clearer multiple peaks (Fig. 8C and D) than iGluSnFR responses. The ranges of numbers of components and open probabilities determined from global fitting of individual synapses were slightly narrower for SnFR-y2 than for iGluSnFR (Fig. 8F \& G).

In summary, the results from the SnFR-y2 sensor were more convincingly quantal. Yet global fitting allowed similar, if less consistent, quantal parameters to be determined from iGluSnFR responses. The ROIs for iGluSnFR were on average much larger in size and number, and a larger fraction of them needed to be manually discarded because the showed strong rundown. A greater proportion of ROls that were responsive for SnFR-Y2 could be described by quantal parameters, and very few were affected by rundown that was seen in $\sim 50 \%$ of the responsive ROls in iGluSnFR-infected neurons.

\section{Discussion}

The principal advantage of optical reporting of neurotransmission is direct access to individual synaptic responses. Taking optical responses from the field of view as group, SnFR- $\gamma 2$ (but not iGluSnFR) accurately reported short term depression, as measured simultaneously with electrophysiology, except in the noisy conditions of $0.5 \mathrm{mM}$ calcium. On average, SnFR-Y2 also gave similar quantal parameters from global estimates of the coefficient of variation (CV) to previous electrophysiological work $\left(N=5\right.$ and $P_{\mathrm{R}}=0.4$ from CV analysis on pairs of cultured rat neurons (Bekkers and Stevens, 1990). These observations suggest that SnFR-Y2 is a fair reporter of presynaptic release, and that post-synaptic short-term plasticity (for example, from desensitization) is limited enough to be neglected at the frequencies we used (up to $5 \mathrm{~Hz}$ ) (Malagon et al., 2016). However, both iGluSnFR and SnFR-Y2 failed to report gross variation in synaptic release properties across individual cells. Are synapses then truly variable (Rosenmund et al., 1993)? The deviation of individual mean/SD values from responsive ROls (stemming from distinct synapses, either neighbouring groups or individual sites) from the fitted line representing the global CV was limited, and the $R^{2}$ value was quite high for all conditions. However, the range of putative maximum release probabilities detected in individual cells was substantial (typically from 
$40-80 \%$, at $4 \mathrm{mM}$ calcium). One caveat is that our approach may be biased towards detecting synapses with higher release rates. Correlation of these values to synaptic architectures, synapses altered by plasticity or experience and synapses whose properties are altered by pathological mutants, should yield information about cell-autonomous synaptic diversity (Matz et al., 2010).

Unlike at the neuromuscular junction (DEL CASTILLO and KATZ, 1954), peaks in the histograms of evoked EPSC amplitudes are much less prominent at central synapses (Bekkers, 1994). Several mathematical treatments were proposed to deal with the smeared distribution that apparently comes from quantal variability at central synapses, including using Bayesian statistics and the gamma distribution (Bhumbra and Beato, 2013; Soares et al., 2019). Optical report of glutamate concentration signal eliminates postsynaptic receptors as a source of variability. Miniature currents are variable in amplitude, introducing unavoidable variance when measuring evoked synaptic currents or when indirectly reporting activity from NMDA receptor calcium flux (Oertner et al., 2002). Simulations suggest that most variability comes from the variation in glutamate released by each vesicle (Franks et al., 2003), but we saw little evidence for this. Perhaps SnFR-Y2 is saturated by even poorly-loaded vesicles, but fitting distributions with release sites $(N)$ up to 8 or more, corresponding to up to 8 quanta in high $\mathrm{Ca}^{2+}$, suggests this is not the case. AMPA-type glutamate receptors have a lower affinity for glutamate than SnFR-Y2, and so the invariant quantal size reported by SnFR-Y2 would in any case translate to less robust AMPA receptor activation. Synaptic AMPARs are probably often not saturated due to a steep dependence on geometric factors (Savtchenko and Rusakov, 2014), or because the number of AMPA receptors available is so variable. Similar lack of dips have been observed in distributions from iGluSnFR in postsynaptic neurons (Soares et al., 2019; Heck et al., 2019), requiring extensive mathematical modelling to extract quantal parameters. In clear contrast, confining iGluSnFR to the presynaptic terminal (where anatomy allows) gives quantal amplitude distributions (Duerst et al., 2020; Dürst et al., 2019). In contrast with some previous work, for example using field stimulation (Heck et al., 2019), in our experiments in autapses, we stimulated glutamate release with a single action potential and obtained quantal histograms with separated peaks. In particular, we can confirm that changing the calcium concentration and examining the same terminal appears to be beneficial in determining quantal parameters (Duerst et al., 2020). On the other hand, in native tissue, the environment of synaptic terminals may reduce the ability of SnFR-Y2 to report quantal distributions, and future work should address this point.

Single AP stimulation enabled a clear view of the systematic fast loss of the iGluSnFR signal (Fig. 8). Why does iGluSnFR run down and why does SnFR-y2 instead have a stable response? One possibility is that bleaching reduces $\Delta F / F$ because a large proportion of the background 
A

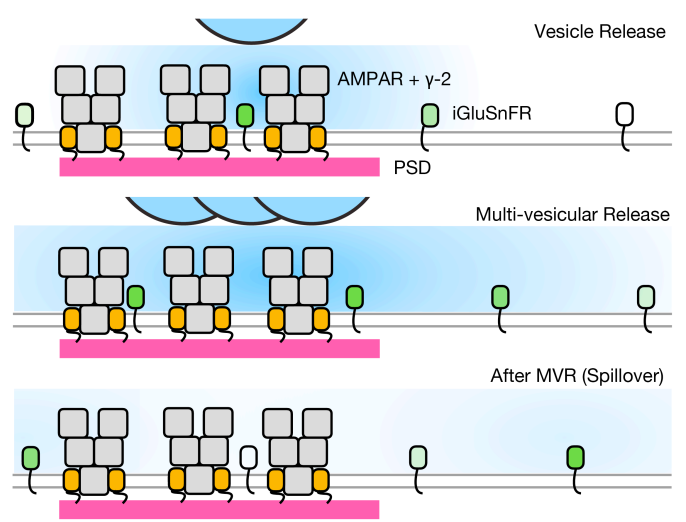

iGluSnFR (Gespons

0000

0000
B SnFR-Y2
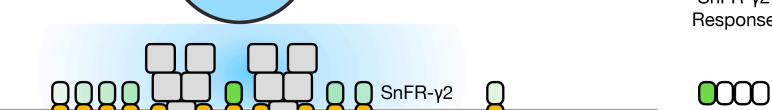

gog

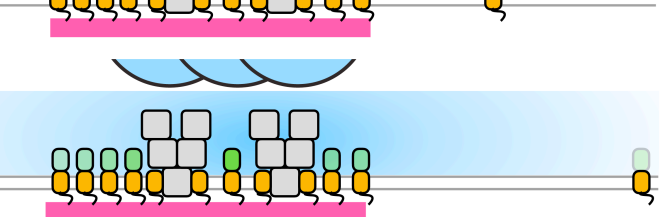

0000

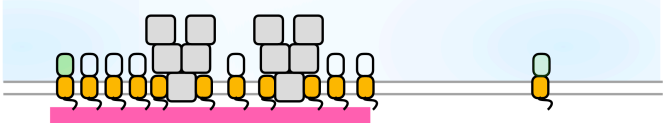

Figure 9 Factors affecting the responses of iGluSnFR and SnFR- $\gamma 2$ (A) Three scenarios following glutamate release from vesicles (blue). iGluSNFR is not localised to the postsynaptic density (PSD, magenta) opposite release sites, where AMPA receptors are docked through their auxiliary proteins like $\gamma-2$ (orange). Therefore iGluSnFR integrates a wide glutamate signal (green response) because of its relatively high affinity for glutamate. This summation gives a larger amplitude signal, and probably incorporates a robust response to extrasynaptic spillover after MVR. Note, in a fluorescence micrograph, the point spread function is larger than the scene depicted here. Also, the camera integration time is longer than the interval between MVR and spillover. These do not provide an obvious explanation for the rundown of SnFR responses. (B) Assuming that SnFR- $\gamma 2$ is concentrated at synapses, and less concentrated outside of synapses, the principal contribution following release of either one vesicle or multiple vesicles (MVR), is from synaptic sites. This limits the signal in amplitude, in space and in time. SnFR- 22 probably competes with postsynaptic AMPA receptors for sites in the PSD.

fluorescence signal comes from either intracellular or occluded sensor molecules. If bleaching (or another process that eliminates signal, like endocytosis) were more intense when glutamate is bound, and iGluSnFR were to in turn have more average occupancy by glutamate than the confined SnFR-Y2, this could specifically reduce $\Delta F$. In such a scheme, SnFR-Y2 would be protected from glutamate exposure by seeing only fast glutamate transients that saturate the sensor and immediately disperse, as opposed to a larger iGluSnFR capacity that is saturated for longer periods by spillover (Fig. 9). The diffusion within synapses of the same single TM protein with and without a PSD binding ligand (from the Stg C-terminus) provides an excellent comparison for our purposes (Li and Blanpied, 2016). These studies show that even though the diffusion of any protein is impeded within the PSD, the membrane protein without any PSD ligand is not excluded. Assuming equal expression, and the concentration of membrane proteins with a PDZ ligand inside PSDs (Li and Blanpied, 2016), the density of SnFR-y2 must be less outside the synapse (also see Fig.6) . On average, a greater capacity outside the synapse would mean that, without being excluded from synapses, iGluSnFR is biased against measuring synaptic glutamate. The basal response of iGluSnFR might be greater in neurons (even though in HEK cells the response was similar) because it is always moving in relation to release sites, and can respond to distant glutamate better by covering greater areas (even within a region of interest). 
Neither iGluSnFR nor SnFR-Y2 can identify single release events in our conditions, but for different reasons. The iGluSnFR signal often runs down intensely, meaning that the quantal estimate $\Delta F$ changes during a recording and cannot be considered constant across different conditions. SnFRY2 cannot reliably report isolated single release events because the amplitude of the signal is too small. However, spontaneous neurotransmission in cultures (in the absence of TTX) could be readily resolved by SnFR-Y2, at spot-like ROls. These optical responses may correspond to single or multiple vesicles released by action potentials. For short term plasticity, whereby synapses are reactivated over $50 \mathrm{~ms}$ intervals or similar, the improved spatial profiles of SnFR-Y2 are expected to be beneficial (Helassa et al., 2018). In our experiments, we did not focus on regions of interest with high-frame rate, but this is an obvious future application.

iGluSnFR has been used in a variety of organisms (James et al., 2019; Marvin et al., 2013; Borghuis et al., 2013), and here we expressed SnFR-Y2 only in cultured rodent neurons. Using mouse auxiliary proteins to anchor the glutamate reporter opposite to release sites may not be universal, and may influence synaptic transmission in other systems in undesirable ways. Stargazin overexpression reduced miniature current amplitude and frequency but did not affect paired pulse release (Fig. S5) and glutamate release was intact (from SnFR-Y2 signals). This strongly suggests a postsynaptic deficit in cultured hippocampal cells but in other neuronal types, effects might be different. When we made inadvertent recordings of GABAergic cells, which we subsequently discarded, we saw no response of the iGluSnFR sensors, but nor was there any obvious effect on evoked inhibitory current magnitudes. It is unclear how well this approach can translate across species, even though PSD binding should be conserved.

Why does overexpressing various forms of Stargazin in the first few days of culture create a specific postsynaptic deficit? Stargazin has a very long first intron (Letts et al., 1998). This long first intron of Stg is the target of three mutations (including the original stargazer mouse) which reduce or abolish y-2 mRNA (Letts et al., 2003). Because long introns extend transcription times, they are thought to delay expression. The most curious aspect of these observations was that release was apparently normal, and presynaptic VGLUT1 markers were not affected. It is not routine to perform electrophysiological characterisation of synapses following molecular interventions before imaging, however, the synapses in neurons infected with Stargazin or chimeras on or before DIV3 were non-functional, despite their normal appearance. It is likely that Stargazin is not the ideal host for the iGluSnFR sensor domain. Auxiliary proteins that are not complexed to AMPA receptors probably fill slots in the postsynaptic density, reducing postsynaptic currents. The performance of the SnFR-ү8 construct was similar to SnFR-ץ2, but neuronal labelling was often less broad, giving fewer ROIs that responded. The additional transmembrane helix from NETO2 appears to reduce expression (although this may indeed be desirable) and is quantitatively more damaging to 
synaptic transmission than Stargazin in a more native form (Fig. 4). Rather than obtaining an optimised sensor, we have instead demonstrated further proof of principle that postsynaptic tethering improves reporter characteristics (Soares et al., 2019), even if it reduces overall signal. Various approaches are known to augment the signal from iGluSnFR including using the superfolder variant of GFP (Marvin et al., 2018), mutagenesis (Fig. S1) or a different promoter (we used human Synapsin). Other tethers, either minimal ones (Li and Blanpied, 2016) or those based on other synaptic proteins, may also give better more defined, or even orthogonal signals.

\section{Materials and Methods}

\section{Materials}

All chemicals were purchased from Sigma-Aldrich unless otherwise stated. MEM Eagle was from PAN-Biotech. FBS, trypsin, and penicillin/streptomycin, Neurobasal-A, B27, Glutamax and gentamicin were from ThermoFisher. All DNA restriction enzymes and T4 ligase were from ThermoFisher. dNTP set was from Qiagen. Plasmid purification kits were from ROBOKLON.

\section{cDNA Constructs and Molecular biology}

pCMV(MinDis).iGluSnFR was a gift from Loren Looger (Addgene plasmid \# 41732 ; http://n2t.net/ addgene:41732 ; RRID:Addgene_41732) (Marvin et al., 2013). pmScarlet_C1 was a gift from Dorus Gadella (Addgene plasmid \# 85042 ; http://n2t.net/addgene:85042 ; RRID:Addgene_85042) (Bindels et al., 2017). pAAV-CW3SL-EGFP was a gift from Bong-Kiun Kaang (Addgene plasmid \# 61463 ; http://n2t.net/addgene:61463 ; RRID:Addgene_61463). mEos3.2-Homer1-N-18 was a gift from Michael Davidson (Addgene plasmid \# 57461 ; http://n2t.net/addgene:57461 ; RRID:Addgene_57461). CMV::SypHy A4 was a gift from Leon Lagnado (Addgene plasmid \# 24478 ; http://n2t.net/addgene:24478 ; RRID:Addgene_24478). GluA2 in the pRK5 vector was the kind gift of Peter Seeburg and Mark Mayer. Stargazin, NETO2 and gamma-8 were kind gifts from Susumu Tomita and Roger Nicoll.

Mutations (including Y230F, Fig. S1) were introduced by overlap PCR to generate iGluSnFR variants. For generation of SnFR-Y2 and SnFR-y8, the Myc tag and PDGFR transmembrane domain were removed from iGluSnFR and replaced by a 12 amino acid linker (GGRARADVYKRQ)

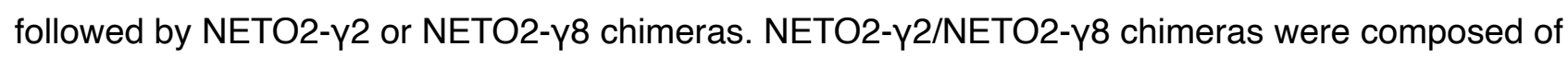
a 79 amino acid stretch of rat NETO2 including the TM segment (starting with residue E333 of Uniprot C6K2K4) and rat Stargazin/gamma-8 with a 3 amino acid (AGS) linker between the two. The entire cassette was subcloned into the pcDNA3.1+ vector. For overlapping PCR of recombinant SnFR-y2/SnFR-y8, we used forward primer 5' CATTGACGCAAATGGGCGGTAG 3' and reverse primer 5' CCGGGCGCGCCCACCTTTCAGTGCCTTGTCATTCGG 3' for the iGluSnFR fragment, and forward primer 5' CCGAATGACAAGGCACTGAAAGGTGGGCGCGCCCGG $3^{\prime}$ and reverse primer 5' CAACAGATGGCTGGCAACTA 3' for NETO2_Y-2/NETO2_Y-8 fragments. To make the mScarlet- 
NETO-Stg construct we replaced the iGluSnFR domain (Glt1 \& cpGFP) in SnFR-Y2 with mScarlet, retaining the $\operatorname{lgK}$ signal to ensure extracellular topology of the $\mathrm{N}$-terminus. For the mScarlet-Stg construct we added mScarlet at the N-terminus of Stargazin. For overlapping PCR of recombinant mScarlet-stg or mScarlet-NETO2-stg, we used forward primer 5' CATTGACGCAAATGGGCGGTAG 3' and reverse primer 5' CTTATACACATCTGCCCGGGCGCGCCCACCCTTGTACAGCTCGTC 3' for the mScarlet fragment, and for Stargazin or NETO2-stargazin fragments we used forward primer 5' ACGAGCtgtacAagGGTGGGCGCGCCCGGGCAGAtGTGtATAAGAGACA 3' and reverse primer 5' CAACAGATGGCTGGCAACTA 3'. For electrophysiological studies on HEK cells we used the pRK5 expression vector encoding the flip splice variant of the rat GluA2 subunit containing a $Q$ at the $Q$ / R-filter, and removed the IRES-eGFP that followed the GluA2 cDNA. The following plasmids are deposited at Addgene: SnFR-gamma2 (Addgene ID: 165495), SnFR-gamma8 (165496), pAAVsyn-SnFR-gamma2-minWPRE (165497), pAAV-syn-SnFR-gamma8-minWPRE (165498).

\section{Lentiviral and Adeno-associated constructs and virus production}

Expression vectors for proteins of interest were delivered to primary neurons by either lentivirus or adeno-associated viruses (AAV). The lentivirus construct for $f(s y n)$-Homer1-tdtomato (BL-1034 in the Charité VCF catalog) was a subclone of mEos3.2-Homer1-N-18. The lentivirus construct for rat Synaptophluorin (Granseth et al., 2006) was a subclone of Addgene \#24478 (BL-0047 in the Charité VCF catalog). For AAV production, each expression cassette (iGluSnFR, iGluSnFR Y230F, SnFR-NETO2-ү2, SnFR-NETO2-ү8, IgK-mScarlet-NETO2- 2 or mScarlet-ү2) was subcloned into a pAAV-backbone with a WPRE-enhanced sequence (Choi et al., 2014) and a synapsin promoter. Preparation of lentiviral particles and AAV were performed by the Charité Viral Core Facility (Charité-Universitätsmedizin, Berlin). Lentiviral particles were prepared as previously described (Lois et al., 2002). Briefly, HEK293T cells were co-transfected with the lentivirus shuttle vector $(10 \mu \mathrm{g})$ and two helper plasmids, PCMVdR8.9 and pVSV.G (5 $\mu \mathrm{g}$ each) using polyethylenimine (PEI). After 72 hours, virus-containing supernatant was collected, filtered, aliquoted and flash-frozen with liquid nitrogen. Virus aliquots were stored at $-80^{\circ} \mathrm{C}$. For infection, about $5 \times 10^{5}$ to $1 \times 10^{6}$ infectious virus units were pipetted onto WT hippocampal neurons per 35 mm-diameter well. AAV were prepared as described previously (Rost et al., 2015).

\section{Mammalian Cell Culture and Transfection}

HEK293 cells for electrophysiological experiments were cultured in Minimum Essential Medium (MEM) supplemented with $10 \%$ (vol/vol) serum, $5 \% \mathrm{U} / \mathrm{mL}$ penicillin and $100 \mu \mathrm{g} / \mathrm{mL}$ streptomycin at $37^{\circ} \mathrm{C}$ in a humidified $5 \% \mathrm{CO} 2$ environment. HEK293 cells were transiently transfected using PEI in a $1: 3$ ratio (v/v; DNA/PEI) with OptiMEM one day after cells were seeded. The ratios for cotransfection were 1:1 for GluA2 and iGluSnFR, 1:2 for GluA2 and SnFR-y2, and 1:5 for GluA2 and SnFR-Y8, up to $3 \mu \mathrm{g}$ total DNA per $35 \mathrm{~mm}$ dish. After 5 hours of incubation, the transfection 
medium was replaced by fresh MEM supplemented with $40 \mu \mathrm{M}$ NBQX with the aim of reducing $\operatorname{TARP}(\mathrm{Y}-2 / \mathrm{Y}-8)$-induced cytotoxicity.

\section{Neuronal Culture and Transduction}

For primary bulk culture of hippocampal neurons, hippocampi were dissected from 1-3 day-old rat brains, cut into small pieces in the dissection medium (Hank's modified solution with $4.2 \mathrm{mM}$ $\mathrm{NaHCO}_{3}, 12 \mathrm{mM}$ HEPES, $33 \mathrm{mM}$ D-Glucose, $200 \mu \mathrm{m}$ Kynurenic acid, $25 \mu \mathrm{m}$ APV, $5 \mu \mathrm{g} / \mathrm{mL}$ Gentamicin, $0.3 \mathrm{mg} / \mathrm{mL} \mathrm{BSA}, 12 \mathrm{mM} \mathrm{MgSO}_{3}, \mathrm{pH} 7.3$ ) and digested in the digestion medium (137 $\mathrm{mM} \mathrm{NaCl}, 5 \mathrm{mM} \mathrm{KCl}, 7 \mathrm{mM} \mathrm{Na} 2 \mathrm{HPO}_{4}, 25 \mathrm{mM}$ HEPES, $4.2 \mathrm{mM} \mathrm{NaHCO}_{3}, 200 \mu \mathrm{m}$ Kynurenic acid, $25 \mu \mathrm{m}$ APV, $0.25 \%$ Trypsin and $0.075 \%$ DNAse, $\mathrm{pH} 7.4$ ) for $5 \mathrm{~min}$ at $37^{\circ} \mathrm{C}$, and then blocked with the dissection medium containing $0.1 \%$ Trypsin inhibitor for $10 \mathrm{~min}$ at $4{ }^{\circ} \mathrm{C}$ and dissociated with the dissociated medium (50 ml Neurobasal-A, $0.5 \mathrm{ml}$ Glutamax 100x, $2 \mathrm{ml} \mathrm{B27} 0.625 \mathrm{ml} \mathrm{FBS}$ ) to acquire isolated neurons. Cultured neurons were incubated at $37^{\circ} \mathrm{C}, 5 \% \mathrm{CO} 2$ in a culture medium composed of Neurobasal-A containing 2\% B27, Glutamax (10 mM) and Gentamycin (0.5 $\mu \mathrm{M})$. Primary hippocampal neurons and autapses were transduced with AAV2/9.iGluSnFR, AAV2/9.iGluSnFR-Y230F, AAV2/9.SnFR-y2, AAV2/9.SnFR-y8, AAV2/9.mScarlet-y2 or AAV2/9.mScarlet-NETO2-y2 at either early (DIV1-3) or late (DIV6) time points. Lentiviruses encoding homer1-tdTomato were added to primary neuronal cultures between 1-3 DIV.

\section{Patch-Clamp Fluorometry on HEK Cells}

Patch-clamp recordings of HEK cells co-expressing glutamate receptors and SnFR/SnFR-y2/ SnFR-Y8 were performed 2-3 days after transfection. Whole HEK293 cells were lifted into the outflow of a piezo-driven fast perfusion switcher for activation by glutamate. For whole-cell patchclamp recordings, the external solution was composed as follows (in $\mathrm{mM}$ ): $158 \mathrm{NaCl}, 20 \mathrm{HEPES}, 3$ $\mathrm{KCl}$, and $1 \mathrm{CaCl}_{2}(\mathrm{pH}$ 7.4). The intracellular (pipette) solution contained (in $\mathrm{mM}$ ): $135 \mathrm{KCl}, 20 \mathrm{KF}$, 20 HEPES, $3 \mathrm{NaCl}, 1 \mathrm{MgCl}_{2}$, and 2 EGTA ( $\mathrm{pH}$ 7.4). Pipettes had a resistance of 3-5 $\mathrm{M} \Omega$ when filled with intracellular solution, and we used ISO-type pipette holders (G23 Instruments) to minimise pipette drift. After whole-cell configuration was obtained, cells were held at $-50 \mathrm{mV}$ and the currents were recorded using Axograph $X$ (Axograph Scientific) via an Instrutech ITC-18 D-A interface (HEKA Elektronik). Excitation by a $488 \mathrm{~nm}$ diode laser (iChrome MLE, Toptica Photonics) for GFP was directed through a manual total internal reflection fluorescence (TIRF) input to an Olympus IX81 microscope. We used a 40x Olympus objective (NA 0.6) for all recordings on HEK cells. Fluorescence intensities in response to $488 \mathrm{~nm}$ excitation were recorded sequentially with 20 ms exposure time, without binning, on a Prime 95B CMOS camera (Photometrics). Images were recorded with MicroManager (Edelstein et al., 2014) and analyzed with FIJI (Schindelin et al., 2012). 


\section{Imaging on Rat Primary Hippocampal Neurons}

Rat primary hippocampal culture was imaged on DIV16-18. The external solution for bath perfusion contained (in mM): $145 \mathrm{NaCl}, 2.5 \mathrm{KCl}, 10 \mathrm{HEPES}, 2 \mathrm{CaCl}_{2}, 1 \mathrm{MgCl}_{2}, 10$ Glucose (pH 7.3). Excitation by $488 \mathrm{~nm}$ and $561 \mathrm{~nm}$ lasers (iChrome MLE, Toptica Photonics) for GFP and RFP was directed through a manual total internal reflection fluorescence (TIRF) input to an Olympus IX81 microscope. We used a 100x Olympus TIRF objective (NA 1.49) for all recordings on bulk culture primary hippocampal neurons. Fluorescence intensities in response to $488 \mathrm{~nm}$ and $561 \mathrm{~nm}$ excitation were recorded sequentially with $20 \mathrm{~ms}$ exposure time and $1 \mathrm{x} 1$ binning on a Prime 95B CMOS camera (Photometrics). GFP and RFP emission were split with a H 560 LPXR superflat beamsplitter (AHF, Germany), and recorded on the same frame after passing through ET525/50 $\mathrm{m}$ (GFP) and ET620/60 m (RFP) filters (both Chroma, all mounted within the Optosplit). Exposures were timed to precede the $-80 \mathrm{mV}$ voltage steps. Laser emission and camera exposure were triggered in hardware directly from the digitizer. Images were recorded with MicroManager and analyzed with Fiji as described above.

\section{Hippocampal autaptic neuronal culture}

Animal housing and use were in compliance with, and approved by, the Animal Welfare Committee of Charite Medical University and the Berlin State Government Agency for Health and Social Services (License T0220/09). Newborn C57BLJ6/N mice (P0-P2) of both sexes were used for all the experiments. Primary neurons were seeded on microisland astrocyte feeder layers that were cultured for 2 weeks before the neuronal culture preparation. Astrocytes derived from C57BL/6N mouse cortices (P0-P1) were plated on collagen/poly-d-lysine microislands made on agarose-coated coverslips using a custom-built rubber stamp to achieve uniform size (200 $\mu \mathrm{m}$ diameter).

Brains from wild-type (WT; C57/BL6N; P0-2) animals were removed and placed in $4^{\circ} \mathrm{C}$ cooled Hank's Buffered Salt Solution (HBSS; GIBCO Life Technologies, Germany). Hippocampi were carefully dissected out and placed in Neurobasal-A Medium supplemented with B27, Glutamax, (all from GIBCO Life Technologies), and penicillin/streptavidin (Roche, Germany; full-NBA) at $37^{\circ} \mathrm{C}$ in a heated shaker. Full-NBA was replaced with Dulbecco's Modified Eagle Medium (DMEM; GIBCO), supplemented with $1 \mathrm{mM} \mathrm{CaCl}_{2}$ and $0.5 \mathrm{mM}$ EDTA (enzyme solution), containing papain (22.5 U/mL; CellSystems $\mathrm{GmbH}$, Germany) and incubated for $45-60 \mathrm{~min}$. The digestion was stopped by removing the enzyme solution and replacing it with an inactivating solution of DMEM supplemented with albumin $(2.5 \mathrm{mg} / \mathrm{mL})$ and trypsin-inhibitor $(2.5 \mathrm{mg} / \mathrm{mL}$; both Sigma-Aldrich). The inactivating solution was removed after $5 \mathrm{~min}$, and replaced with fullNBA. Tissue was dissociated mechanically and cells were counted on a Neubauer chamber. Hippocampal neurons were seeded at $3 \times 10^{3}$ cells onto $30 \mathrm{~mm}$ coverslips previously covered with a dotted pattern of microislands of astrocytes for electrophysiological recordings in autaptic 
bioRxiv preprint doi: https://doi.org/10.1101/2021.01.21.427382; this version posted January 21, 2021. The copyright holder for this preprint (which was not certified by peer review) is the author/funder, who has granted bioRxiv a license to display the preprint in perpetuity. It is made available under aCC-BY 4.0 International license.

cultures, and at a density of $100 \times 10^{3}$ cells onto $30 \mathrm{~mm}$ coverslips previously covered with an astrocyte feeder layer for immunocytochemical staining. Neurons were then incubated at $37^{\circ} \mathrm{C}$ and $5 \% \mathrm{CO}_{2}$ for $14-18$ days. 


\section{Immunocytochemistry}

At DIV 12-16, WT hippocampal neurons infected with the different iGluSnFR chimeric constructs were rinsed with PBS and fixed in 4\% w/v PFA in PBS, pH 7.4 for 10 min at room temperature, after which they were washed 3 times in PBS. After fixation, neurons were permeabilized in PBS-Tween 20 (PBS-T), quenched in PBS-T containing glycine, blocked in PBS-T containing $5 \%$ normal donkey-serum and incubated overnight at $4{ }^{\circ} \mathrm{C}$ with chicken monoclonal antibody against Flag M2 (1:2000; Chemicon, AB5543) and guinea pig polyclonal antibody VGLUT 1 (1:4000; Synaptic System, 135304). Primary antibodies were labeled with Alexa Fluor 405 Affinipure donkey anti-chicken IgG and Alexa Fluor 647 Affinipure donkey anti-guinea pig lgG (1:500 each; Jackson ImmunoResearch). Coverslips with the hippocampal cultures were mounted with Mowiol 4-88 antifade medium (Polysciences Europe). Neuronal images were acquired using an Olympus IX81 inverted epifluorescence microscope at $63 \times$ optical magnification with a CCD camera (Princeton MicroMax; Roper Scientific) and MetaMorph software (Molecular Devices).

At least three independent cultures were imaged and analyzed blind for each experiment. All images were acquired using equal exposure times and subjected to uniform background subtraction and optimal threshold adjustment. After background subtraction and threshold adjustment, images were converted to binary using FIJI. Raw values were exported to Prism (GraphPad) for further analyses.

\section{Electrophysiology of autaptic cultures}

Whole-cell voltage clamp recordings were performed on autaptic hippocampal neurons at DIV14-18 at room temperature. Currents were acquired using a Multiclamp 700B amplifier and a Digidata 1440A digitizer (Molecular Devices). Series resistance was set at $70 \%$ and only cells with series resistances $<12 \mathrm{M} \Omega$ were selected. Data were recorded using Clampex 10 software (Molecular Devices) at $10 \mathrm{kHz}$ and filtered at $3 \mathrm{kHz}$. Borosilicate glass pipettes with a resistance around $3 \mathrm{M} \Omega$ were used and filled with an intracellular solution containing the following (in $\mathrm{mM}$ ): $136 \mathrm{KCl}, 17.8$ HEPES, 1 EGTA, 4.6 $\mathrm{MgCl}_{2}, 4 \mathrm{Na}_{2} \mathrm{ATP}, 0.3 \mathrm{Na}_{2} \mathrm{GTP}, 12$ creatine phosphate, and $50 \mathrm{U} / \mathrm{ml}$ phosphocreatine kinase; $300 \mathrm{mOsm}$; pH 7.4. Neurons were continuously perfused with standard extracellular solution including the following (in $\mathrm{mM}$ ): $140 \mathrm{NaCl}, 2.4 \mathrm{KCl}, 10 \mathrm{HEPES}, 10$ glucose, $2 \mathrm{CaCl}_{2}, 4 \mathrm{MgCl}_{2}$; 300 mOsm; $\mathrm{pH}$ 7.4. When using $4 \mathrm{mM} \mathrm{CaCl}_{2}$, we reduced $\mathrm{MgCl}_{2}$ to 2 $\mathrm{mM}$, and in $0.5 \mathrm{mM} \mathrm{CaCl}_{2}$, the $\mathrm{MgCl}_{2}$ concentration was $5.5 \mathrm{mM}$. Changes in divalent concentration were achieved by local perfusion, and the evoked EPSC was used to monitor the change in response over a 2-3 minute equilibration period. In experiments combining electrophysiology and imaging, movies were acquired using an Olympus IX81 inverted epifluorescence microscope at 63× optical magnification with an Andor iXon EM+ camera and iQ 
software. Spontaneous release was measured by recording mEPSC for $30 \mathrm{~s}$ at $-70 \mathrm{mV}$ and for an equal amount of time in $3 \mu \mathrm{M}$ of the AMPA receptor antagonist NBQX to estimate false positives. We calculated the frequency as the difference in frequency between control and NBQX condition, and the amplitude was the difference of the amplitudes, weighted according to the normalised frequency difference. In some cases, the frequency and amplitude measured was similar for control and NBQX conditions (corresponding to few or no minis detected over background). We discarded amplitudes calculated where the frequencies (control vs NBQX) differed by less than $0.1 \mathrm{~Hz}$, where the amplitude in NBQX was not less than that in control or where the frequency of detected events were higher in NBQX. NBQX action was confirmed by the loss of the evoked EPSC. For each cell, data were filtered at $1 \mathrm{kHz}$ and analyzed using template-based miniature event detection algorithms implemented in the AxoGraph $X$ software. Action potential-evoked EPSCs were elicited by $2 \mathrm{~ms}$ somatic depolarization from -70 to $0 \mathrm{mV}$. Short term plasticity was examined by evoking 5 action potentials with 200 ms interval $(5 \mathrm{~Hz})$. Data were analyzed offline using Axograph X (Axograph Scientific).

\section{Fluorescence data analysis}

Background fluorescence signal was measured from $1 \mu \mathrm{m}$ ROI with the smallest fluorescence intensity in each image that did not show any intensity change during recordings. The baseline level was subtracted from each recording.

For automatic selection of ROls that responded to stimulation, and for extraction of fluorescence data for all ROIs, we wrote FIJI scripts that we include in supplementary files. For calculation of fluorescence change $(\Delta F / F)$, the baseline fluorescence $\left(F_{0}\right)$ was defined as the median fluorescence in the period before the response and then subtracted from $j$-th acquisition frame in a set of $n$ frames and divided by $F_{0}$ to covert each trace to units of $\Delta F / F$ according to equation:

$$
[\Delta F / F]_{\mathrm{j}}=\left(F_{\mathrm{j}}-F_{0}\right) / F_{0}, \mathrm{j}=1,2 \ldots n
$$

For short-term plasticity analysis, we designed a program written in PYTHON, SAFT (repository at : github.com/agplested/SAFT). The workflow of SAFT is shown in Fig. S8. Briefly, the baseline was interactively subtracted using the asymmetric least squares smoothing algorithm (Eilers and Boelens, 2005). Peak finding was done using the SciPy-wavelet transform algorithm (Virtanen et al., 2020) and manually curated by the user in the graphical interface. Peak locations from the mean waveform of responses from all identified ROls was used to extract peak responses from all ROls. Regions of interest with low signal to noise or rundown were excluded, where noted. For coefficient of variance analysis, we fit the following formula (Faber and Korn, 1991),

$$
C V=\frac{\sigma}{\mu}=\left[\left(1-P_{R}\right) / n P_{R}\right]^{\frac{1}{2}}
$$


where $\sigma$ was the standard deviation, and $\mu$ was the mean response, and $n$ was the number of release sites. A global fit using the Solver in Excel was performed, allowing a different release probability $\left(P_{\mathrm{R}}\right)$ for each calcium condition.

To fit the peak response histograms from each region of interest, we used a mixed Gaussian model for fitting the width and number of release sites needed. For global fits across conditions, we used optimise SciPy (Virtanen et al., 2020) on a flattened array of peaks amplitudes, assuming that $N$ and $q$ were constant over calcium variation but release was not and depended on release probability $\left(P_{\mathrm{R}}\right.$, binomial model) or release rate $(\lambda$, Poisson model). In the binomial model, the amplitudes of each Gaussian component were determined with the binomial probability mass function (SciPy) according to the release probability, $P_{\mathrm{R}}$, and a common scale factor both of which were optimised to obtain the fit. For the Poisson model the amplitudes of each Gaussian component were determined with the Poisson probability mass function (SciPy) according to the release rate, $\lambda$, and a common scale factor both of which were optimised to obtain the fit. For the Poisson fit, the widths of each Gaussian component increased in proportion. For each fit, the Kolmogorov-Smirnov test was used to determine goodness of fit. We used the function in SciPy (Virtanen et al., 2020) function and determined the necessary cumulative distribution by hand. Best $N$ values were determined based on the K-S probability and checked manually.

\section{Funding}

This work was supported by the ERC grant 647895 "GluActive" (to A.J.R.P.), the Deutsche Forschungsgemeinschaft (DFG, German Research Foundation) under Germany's Excellence Strategy - EXC-2049 - 390688087 (to both C.R. and A.J.R.P.) and Heisenberg Professorship to A.J.R.P. (DFG PL619/7-1).

\section{Author Contributions}

Y.H. performed and analysed electrophysiology and imaging experiments in HEK cells, bulk cultures and autapses. E.T. performed and analysed electrophysiological experiments in autapses and imaging experiments in bulk cultures, B.K. wrote the script to automatically find ROls and analysed data, A.J.R.P. wrote the SAFT suite and analysed data, A.J.R.P. and C.R. supervised the project, Y.H., E.T. and A.J.R.P. wrote the paper, with input from all authors.

\section{Acknowledgements}

We thank Marcus Wietstruk for molecular biology, Ljudmila Katchan for developing the N-terminal extracellular TARP insertion site, Thorsten Trimbuch and the Viral Core Facility of the Charite for virus production, Heike Lerch, Kordelia Hummel and Niccolò Pampaloni for assistance with neuronal cell culture and Berit Söhl-Kielczynski for help with immunocytochemistry. We thank Teresa Giraldez and Melissa Herman for comments on the manuscript. 


\section{References}

Balaji, J., and Ryan, T. A. (2007). Single-vesicle imaging reveals that synaptic vesicle exocytosis and endocytosis are coupled by a single stochastic mode. Proc Natl Acad Sci U S A 104, 20576-20581.

Bekkers, J. M. (1994). Quantal analysis of synaptic transmission in the central nervous system. Curr Opin Neurobiol 4, 360-365.

Bekkers, J. M., and Stevens, C. F. (1990). Presynaptic mechanism for long-term potentiation in the hippocampus. Nature 346, 724-729.

Bhumbra, G. S., and Beato, M. (2013). Reliable evaluation of the quantal determinants of synaptic efficacy using Bayesian analysis. J Neurophysiol 109, 603-620.

Bindels, D. S., Haarbosch, L., van Weeren, L., Postma, M., Wiese, K. E., Mastop, M., Aumonier, S., Gotthard, G., Royant, A., Hink, M. A., and Gadella, T. W. (2017). mScarlet: a bright monomeric red fluorescent protein for cellular imaging. Nat Methods 14, 53-56.

Borghuis, B. G., Marvin, J. S., Looger, L. L., and Demb, J. B. (2013). Two-photon imaging of nonlinear glutamate release dynamics at bipolar cell synapses in the mouse retina. J Neurosci 33, $10972-10985$.

Chanaday, N. L., and Kavalali, E. T. (2018). Optical detection of three modes of endocytosis at hippocampal synapses. eLlfe

Choi, J. H., Yu, N. K., Baek, G. C., Bakes, J., Seo, D., Nam, H. J., Baek, S. H., Lim, C. S., Lee, Y. S., and Kaang, B. K. (2014). Optimization of AAV expression cassettes to improve packaging capacity and transgene expression in neurons. Mol Brain 7, 17.

Cizeron, M., Qiu, Z., Koniaris, B., Gokhale, R., Komiyama, N. H., Fransén, E., and Grant, S. G. N. (2020). A brain-wide atlas of synapses across the mouse lifespan. Science

DEL CASTILLO, J., and KATZ, B. (1954). Quantal components of the end-plate potential. J Physiol 124, 560-573.

Duerst, C., Wiegert, J. S., Schulze, C., Helassa, N., Török, K., and Oertner, T. G. (2020). The vesicular release probability sets the strength of individual Schaffer collateral synapses. Biorxiv

Dürst, C. D., Wiegert, J. S., Helassa, N., Kerruth, S., Coates, C., Schulze, C., Geeves, M. A., Török, K., and Oertner, T. G. (2019). High-speed imaging of glutamate release with genetically encoded sensors. Nat Protoc 14, 1401-1424.

Edelstein, A. D., Tsuchida, M. A., Amodaj, N., Pinkard, H., Vale, R. D., and Stuurman, N. (2014). Advanced methods of microscope control using $\mu$ Manager software. J Biol Methods 1,

Eilers, P. H. C., and Boelens, H. F. M. (2005). Baseline correction with asymmetric least squares smoothing. Leiden University Medical Centre Report 1, 5.

Enoki, R., Hu, Y.-L., Hamilton, D., and Fine, A. (2009). Expression of long-term plasticity at individual synapses in hippocampus is graded, bidirectional, and mainly presynaptic: optical quantal analysis. Neuron 62, 242-253.

Evans, D. A., Stempel, A. V., Vale, R., Ruehle, S., Lefler, Y., and Branco, T. (2018). A synaptic threshold mechanism for computing escape decisions. Nature 558, 590-594.

Faber, D. S., and Korn, H. (1991). Applicability of the coefficient of variation method for analyzing synaptic plasticity. Biophysical journal 60, 1288-1294.

Farsi, Z., Walde, M., Klementowicz, A. E., Paraskevopoulou, F., and Woehler, A. (2021). Single synapse glutamate imaging reveals multiple levels of release mode regulation in mammalian synapses. iScience 24, 101909.

Franks, K. M., Stevens, C. F., and Sejnowski, T. J. (2003). Independent sources of quantal variability at single glutamatergic synapses. J Neurosci 23, 3186-3195.

Gambino, F., Pagès, S., Kehayas, V., Baptista, D., Tatti, R., Carleton, A., and Holtmaat, A. (2014). Sensory-evoked LTP driven by dendritic plateau potentials in vivo. Nature 515, 116-119.

Granseth, B., Odermatt, B., Royle, S. J., and Lagnado, L. (2006). Clathrin-mediated endocytosis is the dominant mechanism of vesicle retrieval at hippocampal synapses. Neuron $51,773-786$. 
Griesinger, C. B., Richards, C. D., and Ashmore, J. F. (2005). Fast vesicle replenishment allows indefatigable signalling at the first auditory synapse. Nature 435, 212-215.

Heck, J., Parutto, P., Ciuraszkiewicz, A., Bikbaev, A., Freund, R., Mitlöhner, J., Andres-Alonso, M., Fejtova, A., Holcman, D., and Heine, M. (2019). Transient Confinement of Cav2.1 Ca²+-Channel Splice Variants Shapes Synaptic Short-Term Plasticity. Neuron 103, 66-79.e12.

Helassa, N., Dürst, C. D., Coates, C., Kerruth, S., Arif, U., Schulze, C., Wiegert, J. S., Geeves, M., Oertner, T. G., and Török, K. (2018). Ultrafast glutamate sensors resolve high-frequency release at Schaffer collateral synapses. Proc Natl Acad Sci U S A 115, 5594-5599.

Isaac, J. T., Hjelmstad, G. O., Nicoll, R. A., and Malenka, R. C. (1996). Long-term potentiation at single fiber inputs to hippocampal CA1 pyramidal cells. Proc Natl Acad Sci U S A 93, 8710-8715. James, B., Darnet, L., Moya-Díaz, J., Seibel, S. H., and Lagnado, L. (2019). An amplitude code transmits information at a visual synapse. Nat Neurosci 22, 1140-1147.

Jontes, J. D., and Smith, S. J. (2000). Filopodia, spines, and the generation of synaptic diversity. Neuron 27, 11-14.

Kim, Y., Lee, U., Choi, C., and Chang, S. (2020). Release Mode Dynamically Regulates the RRP Refilling Mechanism at Individual Hippocampal Synapses. J Neurosci 40, 8426-8437.

Letts, V. A., Felix, R., Biddlecome, G. H., Arikkath, J., Mahaffey, C. L., Valenzuela, A., Bartlett, F. S., Mori, Y., Campbell, K. P., and Frankel, W. N. (1998). The mouse stargazer gene encodes a neuronal Ca2+-channel gamma subunit. Nat Genet 19, 340-347.

Letts, V. A., Kang, M. G., Mahaffey, C. L., Beyer, B., Tenbrink, H., Campbell, K. P., and Frankel, W. N. (2003). Phenotypic heterogeneity in the stargazin allelic series. Mamm Genome 14, 506-513.

Li, T. P., and Blanpied, T. A. (2016). Control of Transmembrane Protein Diffusion within the Postsynaptic Density Assessed by Simultaneous Single-Molecule Tracking and Localization Microscopy. Front Synaptic Neurosci 8, 19.

Lois, C., Hong, E. J., Pease, S., Brown, E. J., and Baltimore, D. (2002). Germline transmission and tissue-specific expression of transgenes delivered by lentiviral vectors. Science 295, 868-872.

Malagon, G., Miki, T., Llano, I., Neher, E., and Marty, A. (2016). Counting Vesicular Release Events Reveals Binomial Release Statistics at Single Glutamatergic Synapses. J Neurosci 36, 4010-4025.

Marvin, J. S., Borghuis, B. G., Tian, L., Cichon, J., Harnett, M. T., Akerboom, J., Gordus, A., Renninger, S. L., Chen, T.-W., Bargmann, C. I., Orger, M. B., Schreiter, E. R., Demb, J. B., Gan, W.-B., Hires, S. A., and Looger, L. L. (2013). An optimized fluorescent probe for visualizing glutamate neurotransmission. Nat Methods 10, 162-170.

Marvin, J. S., Scholl, B., Wilson, D. E., Podgorski, K., Kazemipour, A., Müller, J. A., Schoch, S., Quiroz, F. J. U., Rebola, N., Bao, H., Little, J. P., Tkachuk, A. N., Cai, E., Hantman, A. W., Wang, S. S., DePiero, V. J., Borghuis, B. G., Chapman, E. R., Dietrich, D., DiGregorio, D. A., Fitzpatrick, D., and Looger, L. L. (2018). Stability, affinity, and chromatic variants of the glutamate sensor iGluSnFR. Nat Methods 15, 936-939.

Matz, J., Gilyan, A., Kolar, A., McCarvill, T., and Krueger, S. R. (2010). Rapid structural alterations of the active zone lead to sustained changes in neurotransmitter release. Proc Natl Acad Sci U S A 107, 8836-8841.

McAllister, A. K., and Stevens, C. F. (2000). Nonsaturation of AMPA and NMDA receptors at hippocampal synapses. Proc Natl Acad Sci U S A 97, 6173-6178.

Miesenböck, G., De Angelis, D. A., and Rothman, J. E. (1998). Visualizing secretion and synaptic transmission with pH-sensitive green fluorescent proteins. Nature 394, 192-195.

Namiki, S., Sakamoto, H., linuma, S., lino, M., and Hirose, K. (2007). Optical glutamate sensor for spatiotemporal analysis of synaptic transmission. Eur J Neurosci 25, 2249-2259.

Oertner, T. G., Sabatini, B. L., Nimchinsky, E. A., and Svoboda, K. (2002). Facilitation at single synapses probed with optical quantal analysis. Nature Neuroscience 5, 657-664.

Okumoto, S., Looger, L. L., Micheva, K. D., Reimer, R. J., Smith, S. J., and Frommer, W. B. (2005). Detection of glutamate release from neurons by genetically encoded surface-displayed FRET nanosensors. Proc Natl Acad Sci U S A 102, 8740-8745. 
Popovic, M. A., Carnevale, N., Rozsa, B., and Zecevic, D. (2015). Electrical behaviour of dendritic spines as revealed by voltage imaging. Nat Commun $6,8436$.

Raghavachari, S., and Lisman, J. E. (2004). Properties of quantal transmission at CA1 synapses. J Neurophysiol 92, 2456-2467.

Ribrault, C., Sekimoto, K., and Triller, A. (2011). From the stochasticity of molecular processes to the variability of synaptic transmission. Nat Rev Neurosci $12,375-387$.

Rosenmund, C., Clements, J. D., and Westbrook, G. L. (1993). Nonuniform probability of glutamate release at a hippocampal synapse. Science 262, 754-757.

Rost, B. R., Schneider, F., Grauel, M. K., Wozny, C., G Bentz, C., Blessing, A., Rosenmund, T., Jentsch, T. J., Schmitz, D., Hegemann, P., and Rosenmund, C. (2015). Optogenetic acidification of synaptic vesicles and lysosomes. Nat Neurosci

Sankaranarayanan, S., De Angelis, D., Rothman, J. E., and Ryan, T. A. (2000). The use of pHluorins for optical measurements of presynaptic activity. Biophys $\mathrm{J} 79,2199-2208$.

Savtchenko, L. P., and Rusakov, D. A. (2014). Moderate AMPA receptor clustering on the nanoscale can efficiently potentiate synaptic current. Philos Trans R Soc Lond B Biol Sci 369, 20130167.

Schindelin, J., Arganda-Carreras, I., Frise, E., Kaynig, V., Longair, M., Pietzsch, T., Preibisch, S., Rueden, C., Saalfeld, S., Schmid, B., Tinevez, J. Y., White, D. J., Hartenstein, V., Eliceiri, K., Tomancak, P., and Cardona, A. (2012). Fiji: an open-source platform for biological-image analysis. Nat Methods 9, 676-682.

Scholl, B., Thomas, C. I., Ryan, M. A., Kamasawa, N., and Fitzpatrick, D. (2020). Cortical response selectivity derives from strength in numbers of synapses. Nature

Soares, C., Trotter, D., Longtin, A., Béïque, J. C., and Naud, R. (2019). Parsing Out the Variability of Transmission at Central Synapses Using Optical Quantal Analysis. Front Synaptic Neurosci 11, 22.

Virtanen, P., SciPy, C., Gommers, R., Oliphant, T. E., Haberland, M., Reddy, T., Cournapeau, D., Burovski, E., Peterson, P., Weckesser, W., Bright, J., van der Walt, S. J., Brett, M., Wilson, J., Millman, K. J., Mayorov, N., Nelson, A. R. J., Jones, E., Kern, R., Larson, E., Carey, C. J., Polat, I.., Feng, Y., Moore, E. W., VanderPlas, J., Laxalde, D., Perktold, J., Cimrman, R., Henriksen, I., Quintero, E. A., Harris, C. R., Archibald, A. M., Ribeiro, A. H., Pedregosa, F., and van Mulbregt, P. (2020). SciPy 1.0: fundamental algorithms for scientific computing in Python. Nature Methods 17, 261-272.

Vyleta, N. P., and Jonas, P. (2014). Loose coupling between Ca2+ channels and release sensors at a plastic hippocampal synapse. Science 343, 665-670.

Zhang, W., St-Gelais, F., Grabner, C. P., Trinidad, J. C., Sumioka, A., Morimoto-Tomita, M., Kim, K. S., Straub, C., Burlingame, A. L., Howe, J. R., and Tomita, S. (2009). A transmembrane accessory subunit that modulates kainate-type glutamate receptors. Neuron $61,385-396$. 


\section{List of Supplementary Materials}

Supplementary Figure 1

Supplementary Figure 2

Supplementary Figure 3

Supplementary Figure 4

Supplementary Figure 5

Supplementary Figure 6

Supplementary Figure 7

Supplementary Figure 8

Supplementary Movie 1

Supplementary Movie 2

Supplementary Movie 3

Supplementary Movie 4
A fast iGluSnFR variant.

The fast iGluSnFR mutant doesn't report neurotransmission.

AMPA-SnFR chimera characterisation.

No influence on synapse density or size by SnFR-y2 or SnFR-y8.

Stg knockdown of synaptic currents.

Detailed view of quantal analysis.

Analysis workflow in the SAFT package.

Examples of poor fits to histograms.

Spontaneous responses of iGluSnFR expressed in bulk culture.

Spontaneous responses of SnFR-y2 expressed in bulk culture.

Evoked responses of iGluSnFR expressed in autaptic neuron culture.

Evoked responses of SnFR-y2 expressed in autaptic neuron culture. 